\title{
Article \\ A Novel Epidemic Model Base on Pulse Charging in Wireless Rechargeable Sensor Networks
}

\author{
Guiyun Liu $1,2,+\oplus$, Xiaokai Su ${ }^{2,+}$, Fenghuo Hong ${ }^{2}$, Xiaojing Zhong ${ }^{1,2, *}$, Zhongwei Liang ${ }^{1, *} \oplus$, Xilai Wu ${ }^{2}$ \\ and Ziyi Huang ${ }^{2}$ \\ 1 Guangzhou Industry \& Information Technology Institute for Intelligent Robotic Equipment, \\ Guangzhou University, Guangzhou 510006, China; liugy@gzhu.edu.cn \\ 2 School of Mechanical and Electric Engineering, Guangzhou University, Guangzhou 510006, China; \\ suxkaii@gmail.com (X.S.); hfh15814933585@outlook.com (F.H.); soberlobe@gmail.com (X.W.); \\ hzy1026931135@gmail.com (Z.H.) \\ * Correspondence: zhongxj@gzhu.edu.cn (X.Z.); liangzhongwei@gzhu.edu.cn (Z.L.) \\ + These authors contributed equally to this work.
}

check for updates

Citation: Liu, G.; Su, X.; Hong, F.; Zhong, X.; Liang, Z.; Wu, X.; Huang, $Z$. A Novel Epidemic Model Base on Pulse Charging in Wireless Rechargeable Sensor Networks. Entropy 2022, 24, 302. https:// doi.org/10.3390/e24020302

Academic Editor: Sergio Saponara

Received: 7 January 2022

Accepted: 14 February 2022

Published: 21 February 2022

Publisher's Note: MDPI stays neutral with regard to jurisdictional claims in published maps and institutional affiliations.

Copyright: (c) 2022 by the authors. Licensee MDPI, Basel, Switzerland. This article is an open access article distributed under the terms and conditions of the Creative Commons Attribution (CC BY) license (https:// creativecommons.org/licenses/by/ $4.0 /)$.

\begin{abstract}
As wireless rechargeable sensor networks (WRSNs) are gradually being widely accepted and recognized, the security issues of WRSNs have also become the focus of research discussion. In the existing WRSNs research, few people introduced the idea of pulse charging. Taking into account the utilization rate of nodes' energy, this paper proposes a novel pulse infectious disease model (SIALS-P), which is composed of susceptible, infected, anti-malware and low-energy susceptible states under pulse charging, to deal with the security issues of WRSNs. In each periodic pulse point, some parts of low energy states (LS nodes, LI nodes) will be converted into the normal energy states (S nodes, I nodes) to control the number of susceptible nodes and infected nodes. This paper first analyzes the local stability of the SIALS-P model by Floquet theory. Then, a suitable comparison system is given by comparing theorem to analyze the stability of malware-free $T$-period solution and the persistence of malware transmission. Additionally, the optimal control of the proposed model is analyzed. Finally, the comparative simulation analysis regarding the proposed model, the non-charging model and the continuous charging model is given, and the effects of parameters on the basic reproduction number of the three models are shown. Meanwhile, the sensitivity of each parameter and the optimal control theory is further verified.
\end{abstract}

Keywords: wireless rechargeable sensor network; pulse charging; cyber security; stability analysis; persistence analysis; optimal control

\section{Introduction}

In recent years, wireless sensor networks (WSNs) have become a hotspot causing extensive attention from researchers [1-5]. Sensor nodes with data storage and data transferring functions make up a sensor network. Nodes can monitor the physical environment near them by deploying in an area without manual monitoring. WSNs have a wide range of applications such as secondary agricultural production, ecological monitoring, traffic safety monitoring, healthcare services and military fields [6]. However, because of the vulnerable nature of nodes and the limited battery capacity, security [7] and short life cycle [8] problems remain to be solved.

Researchers have come up with lots of solutions to optimize energy utilization problems. It is noted that the deployment of rechargeable nodes can solve the energy problem more fundamentally. Wireless rechargeable sensor networks (WRSNs) consist of rechargeable sensor nodes. In recent years, lots of research on WRSNs mainly focuses on the charging planning problem and the energy allocation problem [9-11]. However, the network security of WRSNs is rarely studied. Malware can replicate itself. Once implanted 
into the network system, malware will cause information leakage, network interruption and even network breakdown [12].

Since the infection mechanism of disease in the population is almost the same as the transmission mechanism of malware in WSNs, the epidemiological dynamic is generally used in the study of security issues in WSNs. The applications of epidemiological dynamics are mainly the model's stability analysis. Recent related studies are shown in Table 1.

Table 1. Recent related studies on stability analysis of epidemic models in wireless sensor networks (WSNs).

\begin{tabular}{|c|c|c|}
\hline Authors & Model & Goal \\
\hline J.D. Hernández Guillén et al. [13] & $\begin{array}{c}\text { Susceptible-Carrier-Infectious-Recovered- } \\
\text { Susceptible } \\
\text { (SCIRS) }\end{array}$ & $\begin{array}{l}\text { Exploring local and global stability of } \\
\text { malware-free and epidemic points by } \\
\text { analyzing carrier state. }\end{array}$ \\
\hline D.W. Huang et al. [14] & Susceptible-Infected-Patched-Susceptible (SIPS) & $\begin{array}{l}\text { Through the mechanism of patch } \\
\text { injection, analyze local and global } \\
\text { stability of epidemic point. }\end{array}$ \\
\hline G.Y. Liu et al. [15-22] & $\begin{array}{l}\text { Low-Energy-Node (SILS, SILS-P, SISL, SIRS-L, } \\
\text { SIALS, } \Lambda \text { SILRD, SILRD, SI1I2L) models }\end{array}$ & $\begin{array}{l}\text { Through the introduction of low-energy } \\
\text { nodes, analyze local and global stability } \\
\text { of malware-free and epidemic points }\end{array}$ \\
\hline S.G. Shen et al. [23] & $\begin{array}{c}\text { Vulnerable-Compromised-Quarantined- } \\
\text { Patched-Scrapped } \\
\text { (VCQPS) }\end{array}$ & $\begin{array}{l}\text { By analyzing the heterogeneity and } \\
\text { mobility of sensor nodes in the model, } \\
\text { the local and global stability of } \\
\text { malware-free points is explored. }\end{array}$ \\
\hline R.P. Ojha et al. [24] & $\begin{array}{c}\text { Susceptible-Exposed-Infectious-Quarantine- } \\
\text { Recovered-Vaccinated } \\
\text { (SEIQRV) }\end{array}$ & $\begin{array}{c}\text { By introducing analytical quarantine and } \\
\text { inoculation technology, analyze local and } \\
\text { global stability of worm-free points. }\end{array}$ \\
\hline S. Hosseini et al. [25] & $\begin{array}{c}\text { Susceptible-Exposed-Infected-Recovered- } \\
\text { Susceptible with Quarantine and Vaccination } \\
\text { (SEIRS-QV) }\end{array}$ & $\begin{array}{l}\text { Through the diversification of nodes } \\
\text { configuration, analyze local and global } \\
\text { stability of malware-free points. }\end{array}$ \\
\hline
\end{tabular}

However, there are few studies on the application of epidemic dynamics in WRSNs, and there is almost no research on the pulse charging characteristic of WRSNs. Due to the similarity between the propagation characteristic of epidemic disease in the population and the propagation characteristic of malware attack in WRSNs, the application of pulse effect also gives us a novel method to suppress the spreading of malware. Recent related studies are shown in Table 2.

Table 2. Research on application of pulse effect in epidemic models.

\begin{tabular}{|c|c|c|c|}
\hline Authors & Model & Application Area & Goal \\
\hline A. d'Onofrio et al. [26] & $\begin{array}{c}\text { Susceptible-Exposed-Infected- } \\
\text { Recovered } \\
\text { (SEIR) }\end{array}$ & Anthroponosis & $\begin{array}{l}\text { Pulse inoculation and pulse birth were } \\
\text { introduced to analyze the malware-free } \\
\text { periodic solution and stability of the } \\
\text { malware-free periodic solution. Finally, } \\
\text { to prove that PVS (pulse vaccination } \\
\text { strategy) is more effective than other } \\
\text { vaccination strategies. }\end{array}$ \\
\hline D. Zhang et al. [27] & $\begin{array}{l}\text { Susceptible-Infected-Removed } \\
\text { (SIR) }\end{array}$ & Anthroponosis & $\begin{array}{l}\text { Through the impulsive comparison } \\
\text { theorem and analysis technique, prove } \\
\text { the existence and stability of the } \\
\text { malware-free periodic solution. }\end{array}$ \\
\hline
\end{tabular}


Table 2. Cont.

\begin{tabular}{|c|c|c|c|}
\hline Authors & Model & Application Area & Goal \\
\hline Airen Zhou et al. [28] & $\begin{array}{l}\text { Susceptible-Infected-Removed } \\
\text { (SIR) }\end{array}$ & Anthroponosis & $\begin{array}{l}\text { According to impulsive vaccination } \\
\text { occurring at different moments, prove } \\
\text { the existence and stability of } \\
\text { malware-free periodic solution by } \\
\text { using a stroboscopic map. }\end{array}$ \\
\hline D. Yu et al. [29] & $\begin{array}{l}\text { Susceptible-Infected-Vaccinated- } \\
\text { Susceptible } \\
\text { (SIVS) }\end{array}$ & Anthroponosis & $\begin{array}{l}\text { Using the impulsive comparison } \\
\text { theorem and stroboscopic map, prove } \\
\text { the existence and stability of } \\
\text { malware-free periodic solution and } \\
\text { permanence of the disease. }\end{array}$ \\
\hline
\end{tabular}

Considering the periodic inoculation of

Susceptible-Infected--

S.Z. Wang et $\quad$ Quarantined-RemovedSusceptible (SIQRS) the susceptible population, the stability of the malware-free periodic solution and the persistence of the disease were analyzed using the impulsive comparison theorem.

According to birth pulse and impulsive vaccination occurring at different moments, prove the existence and stability of malware-free periodic solution by using the Poincaré map. Through means of the bifurcation theory, discuss the existence of nontrivial periodic solution bifurcated.

Based on the pulse differential equation, immune operation is achieved on the susceptible nodes in pulse mode.

$\begin{array}{ccc}\text { X.M. Wang et al. [32] } & \begin{array}{c}\text { Susceptible-Infected-Removed } \\ \text { (SIR) }\end{array} & \begin{array}{c}\text { Mobile wireless senso } \\ \text { networks (MWSNs) }\end{array}\end{array}$ immunization period of time.

Due to the rechargeable characteristic, the time of the charging behavior relative to the whole process of the spreading of malware [33] is short, and the charging behavior can be thought of as a pulse activity to some extent. The problem of malware spreading under pulse charging is different from that under continuous charging mode [34]. In this paper, inspired by previous works [35] and taking into account the pulse-charging process, a time-delay free model of WRSNs based on pulse charging, SIALS-P (sensitive-infectionanti-malware-low energy sensitive) is proposed. The model reveals the hardware attack process of malware and the pulse charging process in WRSNs. In this paper, we introduce the local and global stability of the malware-free T-period solution of SIALS-P by using stability analysis theory to prove the persistence of malware transmission and propose an optimal impulsive control strategy.

The main contents of this paper are as follows: the modeling of SIALS-P and the proof of the existence of a malware-free equilibrium state and malware-free $T$-period solution will be introduced in Section 2. Section 3 prove the local stability and global stability of the system. Section 4 demonstrates the persistence of disease. Section 5 proposes and proves the optimal impulsive control strategy. Section 6 shows the simulation results. Section 7 is the conclusion of this paper. 


\section{Modeling Analysis}

\subsection{Epidemic Modeling on WRSNS}

WRSNs is composed of randomly distributed rechargeable nodes. The SIALS (susceptible, infected, anti-malware, low-energy, susceptible) model is first introduced here. It is assumed that the number of nodes increases at a rate $\Lambda$, where $\Lambda$ is greater than 0 . The nodes in the network belong to one of six possible compartments. This model describes the relationships among susceptible nodes $(S)$, infected nodes $(I)$, anti-malware nodes $(A)$, low-energy and susceptible nodes $(L S)$, low-energy and infected nodes $(L I)$ and dysfunctional nodes $(D)$. $S$ nodes are vulnerable to malware, and I nodes are the nodes infected by malware nodes. $A$ nodes clear the malware by activating anti-malware; both $L S$ and $L I$ nodes are at low energy levels and remain dormant. $D$ nodes are totally out of function.

According to the knowledge of epidemic dynamics, the epidemiological coefficients of the models are not less than zero [36].

Thus, the dynamical system can be obtained:

$$
\left\{\begin{array}{l}
\frac{d S(t)}{d t}=\Lambda-\left(\alpha_{1} I(t)+\beta_{1}+\mu\right) S(t), \\
\frac{d I(t)}{d t}=\alpha_{1} S(t) I(t)-\left(\alpha_{2}+\beta_{3}+\mu+\alpha\right) I(t), \\
\frac{d A(t)}{d t}=-\left(\beta_{2}+\mu\right) A(t)+\alpha_{2} I(t), \\
\frac{d L I(t)}{d t}=-\mu L I(t)+\beta_{3} I(t) \\
\frac{d L S(t)}{d t}=-\mu L S(t)+\beta_{1} S(t)+\beta_{2} A(t), \\
\frac{d D(t)}{d t}=\mu N(t)+a I(t),
\end{array}\right.
$$

where $\Lambda$ is the birth rate of susceptible nodes, $\alpha_{1}$ is the transmission rate of infected nodes, $\beta_{1}$ is the wastage rate of susceptible nodes becoming low-energy susceptible nodes, $\mu$ is the mortality rate of nodes, $\alpha_{2}$ is the clearance rate of anti-malicious nodes to infected nodes, $\beta_{3}$ is the wastage rate of infected nodes becoming low-energy infected nodes, $\alpha$ is the mortality rate of infected nodes, $\beta_{2}$ is the attrition rate of anti-malicious nodes becoming low-power and susceptible nodes.

We have $N(t)=S(t)+I(t)+A(t)+L S(t)+L I(t)$, so the supplementary equation is:

$$
\frac{d N(t)}{d t}=\Lambda-\mu N(t)-a I(t),
$$

As $t \rightarrow \infty$, the feasible region is governed by $L S(t)=N(t)-S(t)-A(t)-I(t)-$ $L I(t)$ :

$$
\Omega=\left\{(S, A, I, L I) \in R^{4} \mid 0 \leq N \leq \frac{\Lambda-a I}{\mu}\right\} .
$$

\subsection{A Pulse Charging Model}

By introducing the pulse charging into the above SIALS model, SIALS-P can be obtained when $t=n T(n=1,2,3 \ldots)$ :

$$
\left\{\begin{array}{l}
S\left(t^{+}\right)=S(t)+\gamma L S(t) \\
I\left(t^{+}\right)=I(t)+\gamma L I(t) \\
A\left(t^{+}\right)=A(t) \\
L I\left(t^{+}\right)=(1-\gamma) L I(t) \\
L S\left(t^{+}\right)=(1-\gamma) L S(t) \\
N\left(t^{+}\right)=N(t)
\end{array}\right.
$$

where $T$ is the pulse charging period, and $\gamma$ is the charging rate. $n T^{+}$is used to represent the next instant of $n T$; that is, pulse charging is to charge the low-energy nodes $(L S, L I)$ at a series of time points. When $t \neq n T(n=1,2,3 \ldots)$ : 


$$
\left\{\begin{array}{l}
\frac{d S(t)}{d t}=\Lambda-\left(\alpha_{1} I(t)+\beta_{1}+\mu\right) S(t), \\
\frac{d I(t)}{d t}=\alpha_{1} S(t) I(t)-\left(\alpha_{2}+\beta_{3}+\mu+\alpha\right) I(t), \\
\frac{d A(t)}{d t}=-\left(\beta_{2}+\mu\right) A(t)+\alpha_{2} I(t), \\
\frac{d L I(t)}{d t}=-\mu L I(t)+\beta_{3} I(t), \\
\frac{d L S(t)}{d t}=-\mu L S(t)+\beta_{1} S(t)+\beta_{2} A(t), \\
\frac{d N(t)}{d t}=\Lambda-\mu N(t)-a I(t),
\end{array}\right.
$$

The malware-free $T$-period solution is the periodic solution of $T$ that satisfies the above system of equations when $I=0, L I=0$ and $A=0$, where

$$
S(t)+L S(t)=N(t)=N(\infty)=\frac{\Lambda}{\mu}
$$

Thus, combined with Equations (4)-(6), we can obtain:

$$
\left.\begin{array}{l}
\left.\begin{array}{l}
\frac{d S(t)}{d t}=\Lambda-\left(\beta_{1}+\mu\right) S(t), \\
\frac{d L S(t)}{d t}=-\mu L S(t)+\beta_{1} S(t),
\end{array}\right\} t \neq n T(n=1,2,3 \ldots) \\
S\left(t^{+}\right)=(1-\gamma) S(t)+\frac{\Lambda \gamma}{\mu}, \\
L S\left(t^{+}\right)=(1-\gamma) L S(t),
\end{array}\right\} t=n T(n=1,2,3 \ldots)
$$

In the time interval $[n T,(n+1) T]$, the integral of Equation (7) in the period of two concurrent pulses can be obtained:

$$
L S(t)=\frac{\beta_{1} \Lambda}{\left(\beta_{1}+\mu\right) \mu}+\left(L S\left(n T^{+}\right)-\frac{\beta_{1} \Lambda}{\left(\beta_{1}+\mu\right) \mu}\right) e^{-\left(\beta_{1}+\mu\right)(t-n T)},
$$

where $L S(n T)$ is the initial value at the $n$th pulse time. Using the stroboscopic map [37], in other words, the pulse charging cycle is taken as the stroboscope sampling interval. In the $n$th pulse charging cycle, the value of the state variable at the initial moment of the pulse charging cycle is used to represent the value at the end of the pulse charging cycle. Therefore, we have $L S_{n+1}=f\left(L S_{n}\right)$ when $L S_{n+1}=L S\left((n+1) T^{+}\right)$. Thus, the relationship between different cycles can be obtained:

$$
L S_{n+1}=(1-\gamma)\left[\frac{\beta_{1} \Lambda}{\left(\beta_{1}+\mu\right) \mu}+\left(L S_{n}-\frac{\beta_{1} \Lambda}{\left(\beta_{1}+\mu\right) \mu}\right) e^{-\left(\beta_{1}+\mu\right) T}\right],
$$

When the equilibrium state is reached, there is $L S_{n+1}=L S_{n}$ between the two cycles, and the equilibrium state can be obtained:

$$
L S^{*}=\frac{\frac{\beta_{1} \Lambda}{\left(\beta_{1}+\mu\right) \mu}(1-\gamma)\left(e^{\left(\beta_{1}+\mu\right) T}-1\right)}{e^{\left(\beta_{1}+\mu\right) T}-1+\gamma},
$$

When $t \rightarrow \infty$, from Equation (6) we have:

$$
S^{*}=\frac{\Lambda}{\mu}-L S^{*},
$$

The malware-free $T$-period solution can be obtained:

$$
\left\{\begin{array}{c}
\widetilde{L S}(t)=\frac{\beta_{1} \Lambda}{\left(\beta_{1}+\mu\right) \mu}+\left(L S^{*}-\frac{\beta_{1} \Lambda}{\left(\beta_{1}+\mu\right) \mu}\right) e^{-\left(\beta_{1}+\mu\right)(t-n T)} \\
\widetilde{S}(t)=\frac{\Lambda}{\mu}-\widetilde{L S}(t)
\end{array}\right.
$$

where $t \in[n T,(n+1) T] .(\widetilde{S}(t), \widetilde{L S}(t))$ is the malware-free $T$-period solution of Equation (7). 


\section{Stability Analysis}

The basic reproduction number $R_{0}$ is an important parameter in the early stage of malicious virus infection. It represents the expectation of the number of susceptible nodes that can be infected by an infected node in its average infection cycle after an infected node is introduced into the susceptible node. In general, $R_{0}=1$ can be used as a threshold to determine whether malware is dead or not.

Theorem 1. The equilibrium states $S^{*}$ and $L S^{*}$ of Equations (10) and (11) are locally asymptotically stable and globally asymptotically stable if $R_{0}<1$.

Proof of Theorem 1. Taking the equilibrium states $S^{*}$ and $L S^{*}$ as the initial value of Equation (9), we have:

$$
\left|\frac{d f\left(L S_{n}\right)}{d L S}\right|_{L S=L S^{*}}<1
$$

Therefore, the local stability of the equilibrium state $L S^{*}$ of Equation (10) is locally asymptotically stable according to the stability criterion of differential systems [38]. Because $S^{*}=\frac{\Lambda}{\mu}-L S^{*}$, the local stability of the equilibrium state $S^{*}$ is locally asymptotically stable.

The local stability of $S^{*}$ and $L S^{*}$ means the global stability of $S^{*}$ and $L S^{*}$. This implies that the pulse immunization operations produce the sequences of $S_{n}$ and $L S_{n}$ that converge to the equilibrium states $S^{*}$ and $L S^{*}$.

For the stability of a malware-free T-period solution of Equation (7), we conclude the following theorems:

Theorem 2. The malware-free T-period solution $(\widetilde{S}(t), 0,0,0, \widetilde{L S}(t))$ of the system, is locally asymptotically stable if $R_{0}<1$.

Proof of Theorem 2. Let $Q(t)$ be an $n \times n$ matrix. $\Phi(t)$ is the basic solution matrix of the linear differential system $x^{\prime}(t)=Q(t) x(t)$. In addition, let $r(\Phi Q(t))$ be the spectral radius of $\Phi Q(t)$. Using Floquet theory [39], the local stability of the malware-free $T$ period solution $\widetilde{(S}(t), 0,0,0, \widetilde{L S}(t))$ can be proven. Let the disturbance of the malware-free $T$-period solution of the system be:

$$
x(t)=(S(t), I(t), A(t), L I(t), L S(t)),
$$

Through the linear approximation of Equations (4)-(6), we can obtain the following impulsive differential equation:

$$
\left\{\begin{array}{l}
x^{\prime}(t)=Q(t) x(t), \quad t \neq n T, n \in N \\
x\left(t^{+}\right)=P x(t), \quad t=n T, n \in N
\end{array}\right.
$$

where

$$
\begin{gathered}
Q(t)=\left[\begin{array}{cc}
U & B \\
0 & F-V
\end{array}\right], \quad P=\left[\begin{array}{cc}
P 1 & 0 \\
0 & P 2
\end{array}\right], \\
U=\left[\begin{array}{ccc}
-\left(\beta_{1}+\mu\right) & 0 & 0 \\
0 & -\left(\beta_{2}+\mu\right) & 0 \\
\beta_{1} & \beta_{2} & -\mu
\end{array}\right], \quad B=\left[\begin{array}{cc}
-\alpha_{1} S^{*}(t) & 0 \\
\alpha_{2} & 0 \\
0 & 0
\end{array}\right], \\
F=\left[\begin{array}{cc}
\alpha_{1} S^{*}(t) & 0 \\
0 & 0
\end{array}\right], \quad V=\left[\begin{array}{cc}
\alpha_{2}+\beta_{3}+\mu+a & 0 \\
-\beta_{3} & \mu
\end{array}\right], \\
P_{1}=\left[\begin{array}{ccc}
1 & 0 & \gamma \\
0 & 1 & 0 \\
0 & 0 & 1-\gamma
\end{array}\right], \quad P_{2}=\left[\begin{array}{cc}
1 & \gamma \\
0 & 1-\gamma
\end{array}\right]
\end{gathered}
$$


Because $\Phi_{Q}(t)=\left[\Phi_{i_{j}}\right]$ is the basic solution matrix of the linear differential system $x^{\prime}(t)=Q(t) x(t)$, then $\dot{\Phi}(t)=\Phi(t) Q(t)$, where $\Phi(0)=E_{0}$ ( $E_{0}$ is the unit moment matrix). The differential equation is solved, and we have:

$$
\Phi(t)=\left(\begin{array}{cc}
e^{U T} & \Phi_{B}(t) \\
0 & \Phi_{F-V}(t)
\end{array}\right)
$$

when $t=n T$, it is easy to figure out:

$$
\begin{gathered}
P \Phi(t)=\left(\begin{array}{cc}
P_{1} e^{U T} & P_{1} \Phi_{B}(t) \\
0 & P_{2} \Phi_{F-V}(t)
\end{array}\right), \\
P_{1} e^{U T}=\left[\begin{array}{ccc}
e^{-\left(\beta_{1}+\mu\right) T}+\gamma \beta_{1} T e^{-\mu T} & \gamma \beta_{2} T e^{-\mu T} & \gamma e^{-\mu T} \\
0 & e^{-\left(\beta_{2}+\mu\right) T} & 0 \\
(1-\gamma) \beta_{1} T e^{-\mu T} & (1-\gamma) \beta_{2} T e^{-\mu T} & (1-\gamma) e^{-\mu T}
\end{array}\right] . \\
P_{2} \Phi_{F-V}(t)=\left[\begin{array}{cc}
e^{\int_{0}^{T}\left(\alpha_{1} S^{*}(t)-\left(\alpha_{2}+\beta_{3}+\mu+a\right)\right) d t}+\gamma e^{\beta_{3} T} & \gamma e^{-\mu T} \\
(1-\gamma) e^{\beta_{3} T} & (1-\gamma) e^{-\mu T}
\end{array}\right] .
\end{gathered}
$$

Obviously, the Floquet multipliers of Equation (18) are as follows:

$$
\left\{\begin{array}{c}
\omega_{1}=r\left(P_{1} \mathrm{e}^{U T}\right) \\
\omega_{2}=r\left(P_{2} \Phi_{F-V}(t)\right)
\end{array}\right.
$$

According to Floquet theory and pulse differential equation theory [37], the malwarefree $T$-period solution is locally asymptotically stable if $\omega_{i}<1$, where $i=1,2$. It is difficult for us to see the values of $\omega_{1}$ and $\omega_{2}$ directly. Therefore, in the case that $\omega_{1}$ is less than 1 , there exists a threshold, which can be defined as:

$$
R_{0} \triangleq r\left(P_{2} \Phi_{F-V}(t)\right)
$$

According to Floquet theory, when $R_{0}<1$, the malware-free $T$-period solution $(\widetilde{S}(t), 0,0,0, \widetilde{L S}(t))$ of the system is locally asymptotically stable. Locally asymptotic stability is only for the domain where the periodic solutions are small, and the asymptotic stability of solutions with arbitrary initial values in region $\Omega$ will be proven next.

Theorem 3. When $t \rightarrow \infty, S(t) \rightarrow \widetilde{S}(t)$ and $L S(t) \rightarrow \widetilde{L S}(t)$, the malware-free T-period solution of the system is globally asymptotically stable.

Proof of Theorem 3. From Equation (2), we have: $\Lambda-(\mu+a) N(t) \leq \frac{d N(t)}{d t} \leq \Lambda-\mu N(t)$. It follows that

$$
\frac{\Lambda}{\mu+a} \leq \lim _{t \rightarrow \infty} \inf N(t) \leq \lim _{t \rightarrow \infty} \sup N(t) \leq \frac{\Lambda}{\mu}
$$

From Equation (23), we have:

$$
S(t)+L S(t) \leq \lim _{t \rightarrow \infty} \sup N(t) \leq \frac{\Lambda}{\mu}
$$

From Equations (7) and (24), we can obtain:

$$
\begin{aligned}
& \left.\begin{array}{c}
\frac{d S(t)}{d t} \leq \Lambda-\left(\beta_{1}+\mu\right) S(t), \\
\frac{d L S(t)}{d t}=-\mu L S(t)+\beta_{1} S(t) \leq-\left(\mu+\beta_{1}\right) L S(t)+\frac{\beta_{1} \Lambda}{\mu},
\end{array}\right\} t \neq n T, n \in N, \\
& \left.\begin{array}{c}
S\left(t^{+}\right)=(1-\gamma) S(t)+\frac{\Lambda \gamma}{\mu}, \\
L S\left(t^{+}\right)=(1-\gamma) L S(t),
\end{array}\right\} t=n T, n \in N .
\end{aligned}
$$


Consider the following comparison system:

$$
\left.\begin{array}{c}
x_{1}^{\prime}(t)=\Lambda-\left(\beta_{1}+\mu\right) x_{1}(t), \\
x_{2}^{\prime}(t)=-\left(\mu+\beta_{1}\right) x_{2}(t)+\frac{\beta_{1} \Lambda}{\mu},
\end{array}\right\} t \neq n T, n \in N,
$$

Through the comparison theorem of impulsive differential equations [40], it can be obtained that $S(t) \leq x(t)$ and $L S(t) \leq x(t)$. When $t \rightarrow \infty$, we can obtain $x_{1}(t) \rightarrow S(t)$ and $x_{2}(t) \rightarrow L S(t)$. Then, there are $\varepsilon>0$ and $t_{1}>0$. For any time $t>t_{1}$, we have:

$$
\begin{aligned}
& S(t) \leq x_{1}(t)<\widetilde{S}(t)+\varepsilon, L S(t) \leq x_{2}(t)<\widetilde{L S}(t)+\varepsilon,
\end{aligned}
$$

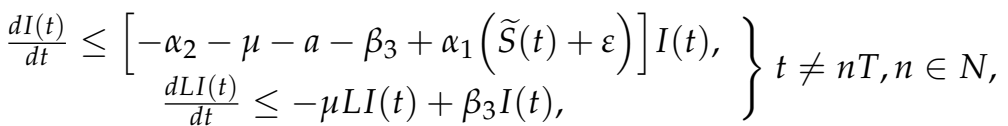

$$
\begin{aligned}
& \left.\begin{array}{l}
I\left(t^{+}\right)=I(t)+\gamma L I(t), \\
L I\left(t^{+}\right)=(1-\gamma) L I(t),
\end{array}\right\} t=n T, n \in N .
\end{aligned}
$$

Suppose $u(t)=\left(\begin{array}{l}u_{1}(t) \\ u_{2}(t)\end{array}\right)$, we have:

$$
\begin{gathered}
(F-V) u(t)=\left(\begin{array}{cc}
-\alpha_{2}-\mu-a-\beta_{3}+\alpha_{1}(\widetilde{S}(t)+\varepsilon) & 0 \\
\beta_{3} & -\mu
\end{array}\right)\left(\begin{array}{l}
u_{1}(t) \\
u_{2}(t)
\end{array}\right) \\
=\left(\begin{array}{c}
{\left[\alpha_{2}-\mu-a-\beta_{3}+\alpha_{1}(\widetilde{S}(t)+\varepsilon)\right] u_{1}(t)} \\
-\mu u_{2}(t)+\beta_{3} u_{1}(t)
\end{array}\right),
\end{gathered}
$$

Consider the following comparison system:

$$
\begin{aligned}
& u^{\prime}(t)=(F-V) u(t), t \neq n T, n \in N, \\
& \left.\begin{array}{c}
u_{1}\left(t^{+}\right)=u_{1}(t)+\gamma u_{2}(t), \\
u_{2}\left(t^{+}\right)=(1-\gamma) u_{2}(t),
\end{array}\right\} t=n T, n \in N .
\end{aligned}
$$

The solution of the system can be expressed as:

$$
u\left(u_{1}, u_{1}\right)=\Phi_{F-V}(t-n T) u\left(n T^{+}\right),
$$

when $t=n T, u\left((n+1) T^{+}\right)=P_{2} \Phi_{F-V}(t-n T) u\left(n T^{+}\right)$. When $t \rightarrow \infty, u_{1} \rightarrow 0$ and $u_{2} \rightarrow 0$. At this time, $\lim _{t \rightarrow \infty} I(t)=0$ and $\lim _{t \rightarrow \infty} L I(t)=0$. Then, there are $\varepsilon_{2}>0$ and $t_{2}>t_{1}$. For any $t>t_{2}$, we have $0<I(t)<\varepsilon_{2}, 0<A(t)<\varepsilon_{2}$ and $0<L I(t)<\varepsilon_{2}$.

Thus, from Equations (7) and (24), we have:

$$
\left.\begin{array}{c}
\Lambda-\left(\alpha_{1} \varepsilon_{2}+\mu+\beta_{1}\right) S(t) \leq \frac{d S(t)}{d t} \leq \Lambda-\left(\mu+\beta_{1}\right) S(t), \\
+\left(\mu+\beta_{1}\right) S(t) \leq \frac{d L S(t)}{d t} \leq-\left(\mu+\beta_{1}\right) L S(t)+\frac{\beta_{1} \Lambda}{\mu}+\beta_{2} \varepsilon_{2},
\end{array}\right\} t \neq n T, n \in N,
$$

Then, consider the following comparison system:

$$
\left.\begin{array}{c}
y_{1}^{\prime} t=\Lambda-\left(\alpha_{1} \varepsilon_{2}+\mu+\beta_{1}\right) y_{1}(t), \\
y_{2}^{\prime} t=-\Lambda+\left(\mu+\beta_{1}\right) S(t),
\end{array}\right\} t \neq n T, n \in N,
$$


Similar to the processing methods for Equations (4) and (5), a set of positive solutions $\widetilde{y}=\left(\widetilde{y_{1}}, \widetilde{y_{2}}\right)$ that are globally asymptotically stable for the comparison system can be obtained, and $\lim _{\varepsilon_{2} \rightarrow 0} \widetilde{y}=(\widetilde{S}(t), \widetilde{L S}(t))$. Through the comparison theorem of impulsive differential equations, we can obtain:

$$
\left\{\begin{array}{c}
y_{1} t<S(t)<x_{1} t \\
y_{2} t<L S(t)<x_{2} t
\end{array}\right.
$$

when $t \rightarrow \infty$, we have:

$$
\left\{\begin{array}{c}
y_{1} \rightarrow \widetilde{y_{1}}, x_{1} \rightarrow \widetilde{S}(t), \\
y_{2} \rightarrow \widetilde{y_{2}}, x_{2} \rightarrow \widetilde{L S}(t),
\end{array}\right.
$$

Then, there is $t_{3}>t_{2}$. For $\varepsilon_{3}>0$, which is small enough, we can obtain when $t>t_{3}$ :

$$
\widetilde{y_{1}}-\varepsilon_{3}<S(t)<\widetilde{S}(t)+\varepsilon_{3}, \widetilde{y_{2}}-\varepsilon_{3}<L S(t)<\widetilde{L S}(t)+\varepsilon_{3} .
$$

Therefore, when $t \rightarrow \infty, S(t) \rightarrow \widetilde{S}(t)$ and $L S(t) \rightarrow \widetilde{L S}(t)$. Therefore, the malwarefree $T$-period solution of the system is globally attractive. That is, after a certain amount of time, the solution is absorbed into a bounded set through motion.

\section{Persistence of Malware Transmission}

In this section, the persistence of malware transmission will be discussed. If certain conditions are met, the malware will not die out; that is, the spread of malware in WRSNs will continue, which is the persistence of malware transmission. The infected nodes ( $I$ noses) are the determinants of the persistence of malware transmission. In order to obtain this result, the following is given:

Lemma 1. When $R_{0}>1$, there is a positive number $\delta$ to make the solution of the SIALS-P to satisfy the following inequality:

$$
\left\{\begin{array}{l}
\limsup _{t \rightarrow \infty} I(t)>\delta \\
\lim _{t \rightarrow \infty} \sup L I(t)>\delta .
\end{array}\right.
$$

Proof of Lemma 1. Using the proof by contradiction, if the above conclusions are not established, there is $t_{1}>0$, and for any $t>t_{1}$, we have $I(t)<\delta$. From Equations (4) and (5), we can obtain:

$$
\left.\begin{array}{c}
\frac{d S(t)}{d t} \geq \Lambda-\left(\alpha_{1} \delta+\beta_{1}+\mu\right) S(t), \\
\frac{d L S(t)}{d t} \geq-\Lambda+\left(\mu+\beta_{1}\right) S(t)+\beta_{2} A(t),
\end{array}\right\} t \neq n T, n \in N,
$$

Consider the following comparison system:

$$
\left.\begin{array}{c}
z_{1}^{\prime} t=\Lambda-\left(\alpha_{1} \delta+\beta_{1}+\mu\right) z_{1}(t), \\
z_{2}^{\prime} t=-\Lambda+\left(\mu+\beta_{1}\right) z_{1}(t)+\beta_{2} A(t),
\end{array}\right\} t \neq n T, n \in N,
$$

From the comparison theorem. We can obtain:

$$
\begin{gathered}
S t \geq z_{1}(t), \\
L S(t) \geq z_{2}(t) .
\end{gathered}
$$


The positive periodic solution $\widetilde{Z}=\left(\widetilde{Z}_{1}, \widetilde{Z}_{2}\right)$ of Equation (39) which is globally stable, can be obtained and $\lim _{\delta \rightarrow 0} \widetilde{Z}=(\widetilde{S}, \widetilde{L S})$. Therefore, there is a positive number $\delta_{1}$ and for any $\varepsilon_{1}>0$ and $\delta<\delta_{1}$, we have $\widetilde{Z}_{1} \geq \widetilde{S}-\varepsilon_{1}$ and $z_{2}^{*} \geq L S^{*}-\varepsilon_{1}$. From the comparison theorem of impulsive differential equations, we have a time variable $t_{2}>t_{1}$ and a positive number $\varepsilon_{2}>0$. For any time $t>t_{2}$, the inequality system is as follows:

$$
\begin{gathered}
S t \geq z_{1}(t) \geq \widetilde{Z}_{1}-\varepsilon_{2} \geq \widetilde{S}-\varepsilon_{1}-\varepsilon_{2}, \\
L S t \geq z_{2}(t) \geq \widetilde{Z}_{2}-\varepsilon_{2} \geq \widetilde{L S}-\varepsilon_{1}-\varepsilon_{2} .
\end{gathered}
$$

The inequality system of Equation (41) is substituted into Equations (4) and (5). We can obtain:

$$
\left.\begin{array}{c}
\frac{d I(t)}{d t} \geq\left[-\alpha_{2}-\mu-a-\beta_{3}+\alpha_{1}\left(S^{*}-\varepsilon_{1}-\varepsilon_{2}\right)\right] I(t), \\
\frac{d L I(t)}{d t} \geq-\mu L I(t)+\beta_{3} I(t), \\
I\left(t^{+}\right)=I(t)+\gamma L I(t), \\
L I\left(t^{+}\right)=(1-\gamma) L I(t),
\end{array}\right\} t=n T, n \in N .
$$

If $\varepsilon_{1}$ and $\varepsilon_{2}$ are small enough to approach 0 , the system can be simplified as:

$$
\left.\begin{array}{c}
\frac{d I(t)}{d t} \geq\left[-\alpha_{2}-\mu-a-\beta_{3}+\alpha_{1} S^{*}\right] I(t), \\
\frac{d L I(t)}{d t} \geq-\mu L I(t)+\beta_{3} I(t), \\
I\left(t^{+}\right)=I(t)+\gamma L I(t), \\
L I\left(t^{+}\right)=(1-\gamma) L I(t),
\end{array}\right\} t=n T, n \in N .
$$

Through the comparison theorem, let $u(t)=\left(\begin{array}{l}u_{1}(t) \\ u_{2}(t)\end{array}\right)$ and construct the following system:

$$
\begin{aligned}
& u^{\prime}(t)=(F-V) u(t), t \neq n T, n \in N, \\
& \left.\begin{array}{c}
u_{1}\left(t^{+}\right)=u_{1}(t)+\gamma u_{2}(t), \\
u_{2}\left(t^{+}\right)=(1-\gamma) u_{2}(t),
\end{array}\right\} t=n T, n \in N .
\end{aligned}
$$

The above system satisfies $u\left(t, n T, u\left(n T^{+}\right)\right)=\phi_{F-v}(t-n T) u\left(n T^{+}\right), u\left((n+1) T^{+}\right)=$ $P_{2} \phi_{F-v}(t-n T) u\left(n T^{+}\right)$. When $R_{0}>1$, as $\mathrm{t} \rightarrow \infty$ and $u_{1} \rightarrow \infty$ and $u_{2} \rightarrow \infty$, we can obtain the conclusion as follows:

$$
\begin{aligned}
& \lim _{t \rightarrow \infty} I(t)=\infty, \\
& \lim _{t \rightarrow \infty} L I(t)=\infty .
\end{aligned}
$$

The above conclusion contradicts the condition $I t<\delta$ and $L I(t)<\delta$ which was established previously. Hence, Lemma 1 is proved.

Through Lemma 1, we can obtain the following theorem:

Theorem 4. When $R_{0}>1$, the malware transmission is uniformly persistent, that is, there is a positive number $\eta$ that makes the solution of the system satisfy the following inequality:

$$
\begin{aligned}
& \liminf _{t \rightarrow \infty} I(t)>\eta, \\
& \lim _{t \rightarrow \infty} \inf L I(t)>\eta .
\end{aligned}
$$

Proof of Theorem 4. According to Lemma 1, there are two possible situations when the malware transmission is uniformly persistent:

(I). When the time variable $T$ is large enough, $I(t)>\eta$ and $L I(t)>\eta$;

(II). When the time variable $T$ is large enough, $I(t)$ and $L I(t)$ oscillate nearby $\eta$. 
If case (I) is true, it is clear that malware transmission is uniformly persistent. Therefore, we focus on case (II). From Lemma 1, we can obtain:

$$
\begin{aligned}
& \limsup _{t \rightarrow \infty} \operatorname{su}(t)>\delta, \\
& \lim _{t \rightarrow \infty} \sup L I(t)>\delta .
\end{aligned}
$$

Therefore, in the case of oscillation, we have:

$$
\begin{aligned}
& \left.\begin{array}{c}
I\left(t_{1}\right) \geq \delta, \\
L I\left(t_{1}\right) \geq \delta,
\end{array}\right\} t_{1} \in\left(n_{1} T,(n+1) T\right], \\
& \left.\begin{array}{r}
I\left(t_{2}\right) \geq \delta, \\
L I\left(t_{2}\right) \geq \delta,
\end{array}\right\} t_{2} \in\left(n_{2} T,(n+1) T\right] .
\end{aligned}
$$

where $n_{2}>n_{1}$ and when $t \in\left[t_{1}, t_{2}\right]$, it can be obtained as follows:

$$
\begin{gathered}
\frac{d L I(t)}{d t}=-\mu L I(t)+\beta_{3} I(t) \geq-\mu L I(t), \quad t \neq n T, n \in N, \\
L I\left(t^{+}\right)=(1-\gamma) L I(t), \quad t=n T, n \in N .
\end{gathered}
$$

We can obtain:

$$
L I(t) \geq L I\left(t_{1}\right) e^{-\mu\left(t-t_{1}\right)} \geq \delta e^{-\mu\left(t-t_{1}\right)} \geq \delta e^{-\mu\left(n_{2}-n_{1}+1\right) T},
$$

Additionally, when $t=n T$, we can obtain:

$$
L I(t) \geq \delta(1-\gamma)^{n_{2}-n_{1}} e^{-\mu\left(n_{2}-n_{1}+1\right) T},
$$

Then, for $I(t)$, we have:

$$
\begin{gathered}
\frac{d I(t)}{d t}=\left[-\alpha_{2}-\mu-a-\beta_{3}+\alpha_{1} S(t)\right] I(t) \geq\left(-\alpha_{2}-\mu-a-\beta_{3}\right) I(t), \\
I\left(t^{+}\right)=I(t)+\gamma L I(t) .
\end{gathered}
$$

By applying the solution method similar to Equation (52), we have:

$$
I(t) \geq \delta e^{\left(-\alpha_{2}-\mu-a-\beta_{3}\right)\left(n_{2}-n_{1}+1\right) T}+\gamma^{n_{2}-n_{1}} \delta(1-\gamma)^{n_{2}-n_{1}} e^{-\mu\left(n_{2}-n_{1}+1\right) T},
$$

Let $\eta_{1}=\min \left\{\delta e^{\left(-\alpha_{2}-\mu-a-\beta_{3}\right)\left(n_{2}-n_{1}+1\right) T}+\gamma^{n_{2}-n_{1}} \delta(1-\gamma)^{n_{2}-n_{1}} e^{-\mu\left(n_{2}-n_{1}+1\right) T}, \delta(1-\gamma)^{n_{2}-n_{1}} e^{-\mu\left(n_{2}-n_{1}+1\right) T}\right\}$.

Due to $n_{2}-n_{1} \geq 0$ and the fact that it is bounded, $\eta_{1}$ cannot be infinitesimal. Hence, we can obtain $I(t) \geq \eta_{1}$ and $L I(t) \geq \eta_{1}$.

For $t>t_{2}$, there is a positive number $\eta_{2}$. Therefore, we can obtain the sequence $\left\{\eta_{j}\right\}, j=1,2, \ldots k \ldots$, where

$$
\begin{gathered}
\eta_{1}=\min \left\{\delta e^{\left(-\alpha_{2}-\mu-a-\beta_{3}\right)\left(n_{2}-n_{1}+1\right) T}+\gamma^{n_{2}-n_{1}} \delta(1-\gamma)^{n_{2}-n_{1}} e^{-\mu\left(n_{2}-n_{1}+1\right) T},\right. \\
\left.(1-\gamma)^{n_{2}-n_{1}} e^{-\mu\left(n_{2}-n_{1}+1\right) T}\right\},
\end{gathered}
$$

When $t \in\left(t_{k}, t_{k+1}\right)$, the inequality can be derived as follows where $t_{k} \in n_{k} T,\left(n_{k}+1\right) T$ and $t_{k+1} \in n_{k+1} T,\left(n_{k+1}+1\right) T$ :

$$
\begin{gathered}
I(t) \geq \eta_{k}>0, \\
L I(t) \geq \eta_{k}>0 .
\end{gathered}
$$

Let $\eta^{*}=\min \eta_{\mathrm{j}}$. For any $t>t_{1}$, there is $I(t) \geq \eta^{*}>0$ and $L I(t) \geq \eta^{*}>0$. Therefore, Theorem 4 is proven. 


\section{Optimal Control}

In the optimal control theory of epidemic dynamics, the primary goal is to minimize the number of infections while minimizing the cost of vaccination [41].

The probability that the infected node becomes the anti-malware node after receiving information from the anti-malware node is $\alpha_{2}$. In the process of constructing the minimum objective function, $\alpha_{2}$ is selected as the control variable that changes over time between the pulse points and is represented by $\alpha_{2}(t)$, and $0 \leq \alpha_{2}(t) \leq 1$. Control variable $\alpha_{2}(t)$ is constantly changing over time. Unlike $\alpha_{2}(t), v(n T)$ is the control variable of the pulse point and a targeted therapy strategy to reduce infected nodes, and $0 \leq v(n T) \leq 1$.

In this section, the ultimate goal of optimal control is to minimize the number of infected nodes and minimize the cost of activating anti-malware and detecting and killing malware. Thus, in the SIALS-P model, we can construct the minimized objective function as:

$$
J\left(g_{i}(t), v_{i}(n T)\right)=\int_{0}^{\delta T}\left(A_{1} I(t)+A_{2} L I(t)+\frac{A_{3}}{2} \alpha_{2}^{2}(t)\right) \mathrm{d} t+\sum_{i=1}^{\eta}\left(\frac{B}{2} v^{2}(n T)\right),
$$

where $\delta T$ represents the duration of optimal control and $\delta \in N . A_{1}$ and $A_{2}$ are the monitoring costs of $I$ nodes and $L I$ nodes, respectively. $A_{3}$ and $B$ are the costs of implementing control strategies.

Introduce control variables $\alpha_{2}$ and $v(n T)$ into Equations (4) and (5). The objective function subject is:

$$
\left\{\begin{array}{l}
\frac{d S(t)}{d t}=\Lambda-\left(\alpha_{1} I(t)+\beta_{1}+\mu\right) S(t), \\
\frac{d I(t)}{d t}=\alpha_{1} S(t) I(t)-\left(\alpha_{2}(t)+\beta_{3}+\mu+\alpha\right) I(t) \\
\frac{d A(t)}{d t}=-\left(\beta_{2}+\mu\right) A(t)+\alpha_{2}(t) I(t), \\
\frac{d L I(t)}{d t}=-\mu L I(t)+\beta_{3} I(t), \\
\frac{d L S(t)}{d t}=-\mu L S(t)+\beta_{1} S(t)+\beta_{2} A(t), \\
S\left(t^{+}\right)=S(t)+\gamma L S(t)+v(n T) I(t), \\
I\left(t^{+}\right)=I(t)+\gamma L I(t)-v(n T) I(t), \\
A\left(t^{+}\right)=A(t), \\
L I\left(t^{+}\right)=(1-\gamma) L I(t), \\
L S\left(t^{+}\right)=(1-\gamma) L S(t),
\end{array}\right\} t=n T, n \in N, n \in N
$$

In order to achieve the optimal control objective, the Hamiltonian $H$ without control as the function is constructed as:

$$
\begin{gathered}
H=A_{1} I(t)+A_{2} L I(t)+\frac{A_{3}}{2} \alpha_{2}^{2}(t)+\lambda_{1}(t) S_{i}(t)+\lambda_{2}(t) I_{i} \dot{(}(t) \\
\left.+\lambda_{3}(t) A(t)+\lambda_{4}(t) L I \dot{(t}\right)+\lambda_{5}(t) L \dot{S}(t)
\end{gathered}
$$

We construct the impulse Hamiltonian function $H^{P}$ defined as:

$$
\begin{gathered}
H^{P}=\frac{B}{2} v^{2}(n T)+\gamma \lambda_{1}\left(n T^{+}\right) L S(t)+v \lambda_{1}\left(n T^{+}\right) I(t)+\gamma \lambda_{2}\left(n T^{+}\right) L I(t) \\
-v \lambda_{2}\left(n T^{+}\right) I(t)-\gamma \lambda_{4}\left(n T^{+}\right) L I(t)-\gamma \lambda_{5}\left(n T^{+}\right) L S(t)
\end{gathered}
$$

We use the Pontryagin maximum principle to obtain the necessary conditions for optimal control.

Theorem 5. Introduce optimal controls $\alpha_{2}^{*}(t)$ and $v^{*}(n T)$, and solutions $S^{*}(t), I^{*}(t), A^{*}(t)$, $L I^{*}(t)$ and $L S^{*}(t)$ into Equation (57). There exist adjoint variables $\lambda_{k}(t), k=1,2,3,4$. 
$t \neq n T$ provides an optimal control $\alpha_{2}^{*}(t)$. The adjoint variables $\lambda_{k}(t)$ satisfy the following adjoint differential system.

$$
\left\{\begin{aligned}
\frac{d \lambda_{1}(t)}{d t} & =-\frac{\partial H}{\partial S}=-\left(-\lambda_{1}(t)\left(\alpha_{1} I(t)+\beta_{1}+\mu\right)+\alpha_{1} \lambda_{2}(t) I(t)+\beta_{1} \lambda_{5}(t)\right), \\
\frac{d \lambda_{2}(t)}{d t} & =-\frac{\partial H}{\partial I}=-\left(A_{1}+\left(\alpha_{1} S(t)-\left(\alpha_{2}(\mathrm{t})+\beta_{3}+\mu+\alpha\right)\right) \lambda_{2}(t)+\alpha_{2}(t) \lambda_{3}+\beta_{3} \lambda_{4}\right), \\
\frac{d \lambda_{3}(t)}{d t} & =-\frac{\partial H}{\partial A}=-\left(-\left(\beta_{2}+\mu\right) \lambda_{3}+\beta_{2} \lambda_{5}\right) \\
\frac{d \lambda_{4}(t)}{d t} & =-\frac{\partial H}{\partial L I}=-\left(A_{2}-\mu \lambda_{4}\right) \\
\frac{d \lambda_{5}(t)}{d t} & =-\frac{\partial H}{\partial L S}=-\left(-\mu \lambda_{5}\right)
\end{aligned}\right.
$$

Transversality conditions are $\lambda_{k}(\delta T)=0$, and the optimal control $\alpha_{2}^{*}(t)$ can be solved as $\frac{\partial H}{\partial \alpha_{2}(t)}=0$. Thus, we have the optimal control of the continuous part as follows:

$$
\alpha_{2}^{*}(t)=\frac{\left(\lambda_{2}(t)-\lambda_{3}(t)\right) I^{*}(t)}{A_{3}}
$$

$t=n T$ provides the impulse optimal control $v^{*}(n T)$. There exist adjoint variables $\lambda_{k}(n T), k=1,2,3,4$ and we have:

$$
\left\{\begin{array}{l}
\lambda_{1}(n T)=\lambda_{1}\left(n T^{+}\right), \\
\lambda_{2}(n T)=\lambda_{2}\left(n T^{+}\right)-v \lambda_{2}\left(n T^{+}\right)+v \lambda_{1}\left(n T^{+}\right), \\
\lambda_{3}(n T)=\lambda_{3}\left(n T^{+}\right), \\
\lambda_{4}(n T)=\lambda_{4}\left(n T^{+}\right)-\gamma \lambda_{4}\left(n T^{+}\right)+\gamma \lambda_{2}\left(n T^{+}\right), \\
\lambda_{5}(n T)=\lambda_{5}\left(n T^{+}\right)-\gamma \lambda_{5}\left(n T^{+}\right)+\gamma \lambda_{1}\left(n T^{+}\right),
\end{array}\right.
$$

The optimality condition at $v(n T)=v^{*}(n T)$ implies that $\frac{\partial H^{P}}{\partial v(n T)}=0$. Therefore, the optimal control in any impulse point is obtained.

$$
v^{*}(n T)=\frac{\left(\lambda_{2}\left(n T^{+}\right)-\lambda_{1}\left(n T^{+}\right)\right) I^{*}(t)}{B},
$$

$S^{*}(t), I^{*}(t), A^{*}(t), L I^{*}(t)$ and $L S^{*}(t)$ are the solutions for Equation (57) to perform optimal control. Let $x(t)=(S(t), I(t), A(t), L I(t), L S(t))$, which is left-continuous on $[0, T]$ and $x_{i}(n T)=x_{i}\left(n T^{-}\right)$. We can solve the optimal level the pulse intensity $v$ when the sequences of impulse point $n T$ are fixed. Let $x(t)=x^{*}(t)$, the optimal controls are as follows:

$$
\alpha_{2}^{*}(t)= \begin{cases}1, & \frac{\left(\lambda_{2}(t)-\lambda_{3}(t)\right) I^{*}(t)}{A_{3}} \geq 1 \\ \frac{\left(\lambda_{2}(t)-\lambda_{3}(t)\right) I^{*}(t)}{A_{3}}, & 0<\frac{\left(\lambda_{2}(t)-\lambda_{3}(t)\right) I^{*}(t)}{A_{3}}<1 \\ 0, & \frac{\left(\lambda_{2}(t)-\lambda_{3}(t)\right) I^{*}(t)}{A_{3}} \leq 0\end{cases}
$$

and

$$
v^{*}(n T)= \begin{cases}1, & \frac{\left(\lambda_{2}\left(n T^{+}\right)-\lambda_{1}\left(n T^{+}\right)\right) I^{*}(t)}{B} \geq 1 \\ \frac{\left(\lambda_{2}\left(n T^{+}\right)-\lambda_{1}\left(n T^{+}\right)\right) I^{*}(t)}{B}, & 0<\frac{\left(\lambda_{2}\left(n T^{+}\right)-\lambda_{1}\left(n T^{+}\right)\right) I^{*}(t)}{B}<1 \\ 0, & \frac{\left(\lambda_{2}\left(n T^{+}\right)-\lambda_{1}\left(n T^{+}\right)\right) I^{*}(t)}{B} \leq 0 .\end{cases}
$$

The optimal control functions can also be simplified as:

$$
\alpha_{2}^{*}(t)=\min \left(\max \left(0, \frac{\left(\lambda_{2}(t)-\lambda_{3}(t)\right) I^{*}(t)}{A_{3}}\right), 1\right),
$$

and

$$
v^{*}(n T)=\min \left(\max \left(0, \frac{\left(\lambda_{2}\left(n T^{+}\right)-\lambda_{1}\left(n T^{+}\right)\right) I^{*}(t)}{B}\right), 1\right) .
$$




\section{Simulation}

The purpose of this section is to further verify the correctness and practicability of our theory by numerical simulation. We compared the relationship between the pulse charging model, the continuous charging model and the non-charging model. Thus, the advantage of pulse charging over the other two models is received. In Section 6.1, we use the Runge-Kutta [42] method to analyze the stability of pulse charging in MATLAB and compare it with the other two models. The effects of various control parameters on the basic reproduction number of three charging models are analyzed in Section 6.1.

\subsection{Stable Analysis When $R_{0}<1$}

This subsection verifies the stability of the basic reproduction number of the three charging models $R_{i}<1(i=0,1,2)$, where, $R_{0}$ is the basic reproduction number of the pulse charging model, $R_{1}$ is the basic reproduction number of the continuous charging model and $R_{2}$ is the basic reproduction number of the non-charging model. Parameters are set as $\Lambda=0.2, \mu=0.004, \beta_{1}=0.005, \beta_{2}=0.005, \beta_{3}=0.008, \alpha_{1}=0.0001, \alpha_{2}=0.001, \gamma=0.05$, $a=0.005, T=10$. It is assumed that $N(t)=S(t)+I(t)+A(t)+L S(t)+L I(t) \leq 50$, $S_{0}=48, I_{0}=2, A_{0}=0, L S_{0}=0$ and $L I_{0}=0$.

Because $R_{0} \triangleq r\left(P_{2} \Phi_{F-V}(t)\right)$, we can obtain $R_{0}=0.4828<1$. Therefore, there will be a malware-free $T$-period solution $(\widetilde{S}, 0,0,0, \widetilde{L S})$. In Figure 1, all nodes will approach stability. When $T$ is approaching infinity, the $I$ and $L I$ nodes are almost zero, while the $S$ and $L S$ nodes will be stable at non-zero values. The basic reproduction number of the other two models are calculated by:

$$
R_{1}=\frac{\alpha_{1} \Lambda(\gamma+\mu)^{2}}{\left[\left(\beta_{1}+\mu\right)(\gamma+\mu)-\gamma \beta_{1}\right]\left[\left(\alpha_{1}+\beta_{3}+\mu+a\right)(\gamma+\mu)-\gamma \beta_{3}\right]},
$$

It is easily obtained that $R_{1}=0.4320<1, R_{2}=0.1481<1$. Thus, the two models both have a global stable malware-free equilibrium point $(S(t), 0,0,0, L S(t))$. As can be seen in Figures 2 and 3, for the continuous charging model and the non-charging model, when $t=1200$, the number of $S$ are 45.72 and 22.22, respectively. Compared with the pulse charging model in Figure $1, S=31.84$ when $t=1200$. It can clearly be seen that the number of $S$ under the pulse charging model is lower than that under the continuous charging model but is higher than that of the non-charged model. However, the relationship among the number of $L S$ is opposite to that among the number of $S$. Therefore, the pulse charge can better improve the utilization rate of energy and the work efficiency of WRSNs.

\subsection{Influence of Parameters on the Basic Reproduction Number}

Parameters are set as $\mu=0.004, \beta_{1}=0.005, \beta_{3}=0.008, \alpha_{1}=0.0001, \alpha_{2}=0.001$, $\gamma=0.05, a=0.005, T=10, \Lambda \in[0,0.4], \beta_{2} \in[0,0.05]$.

As shown in Figure 4, the basic reproduction number will increase with the increase in $\Lambda$. It is obvious that $\beta_{2}$ under the pulse charging model has greater influence than that under the other two models on the basic reproduction number. It can be seen that some values of the basic reproduction number under the pulse charging model are located between those under the other two models. 

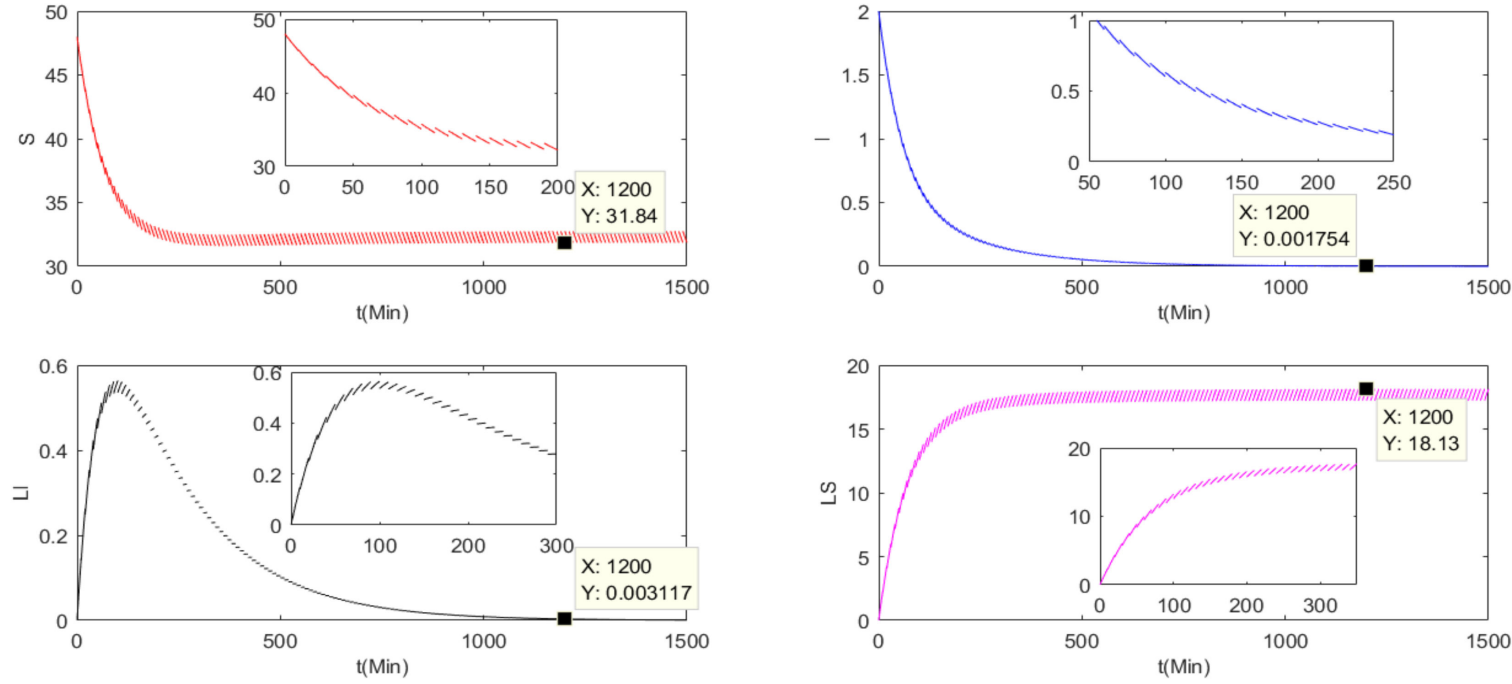

Figure 1. The nodes' numbers under the pulse charging model with $R_{0}<1$.
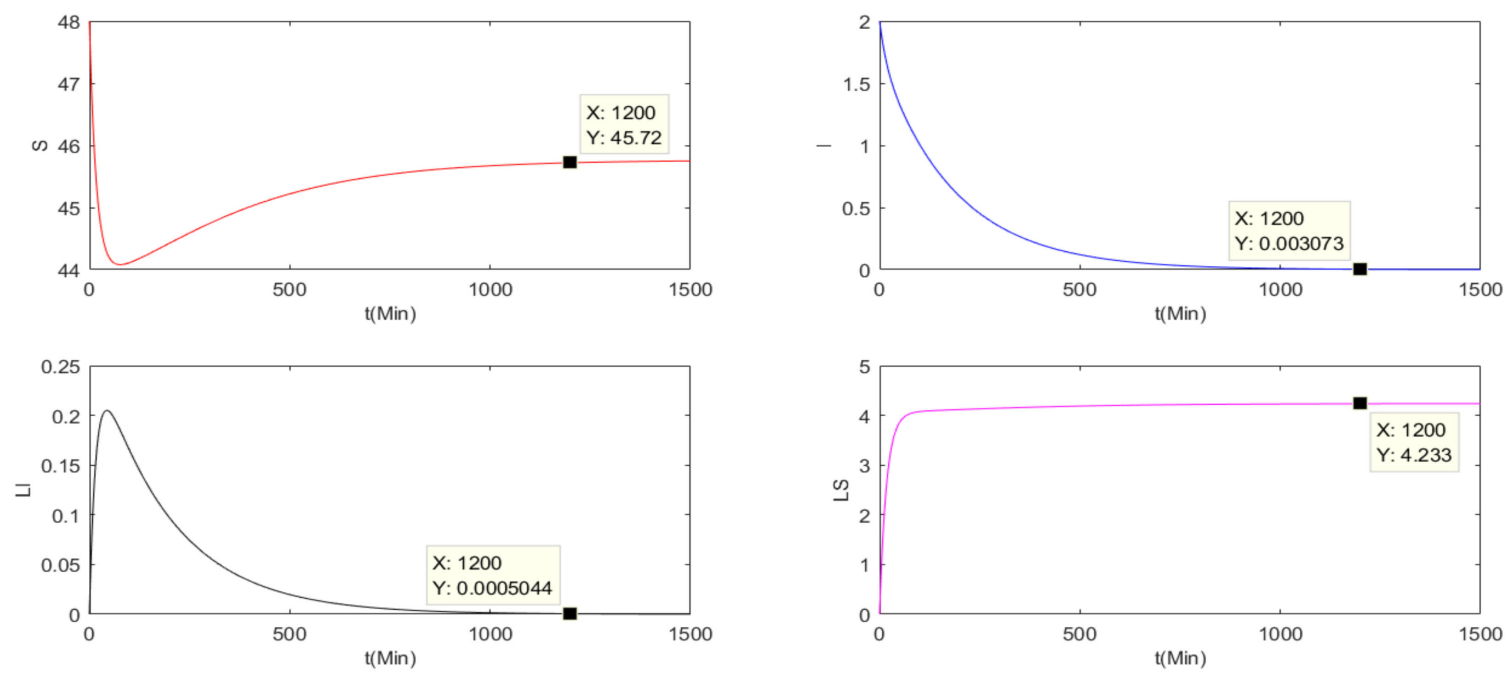

Figure 2. The nodes' numbers under the continuous charging model with $R_{1}<1$.
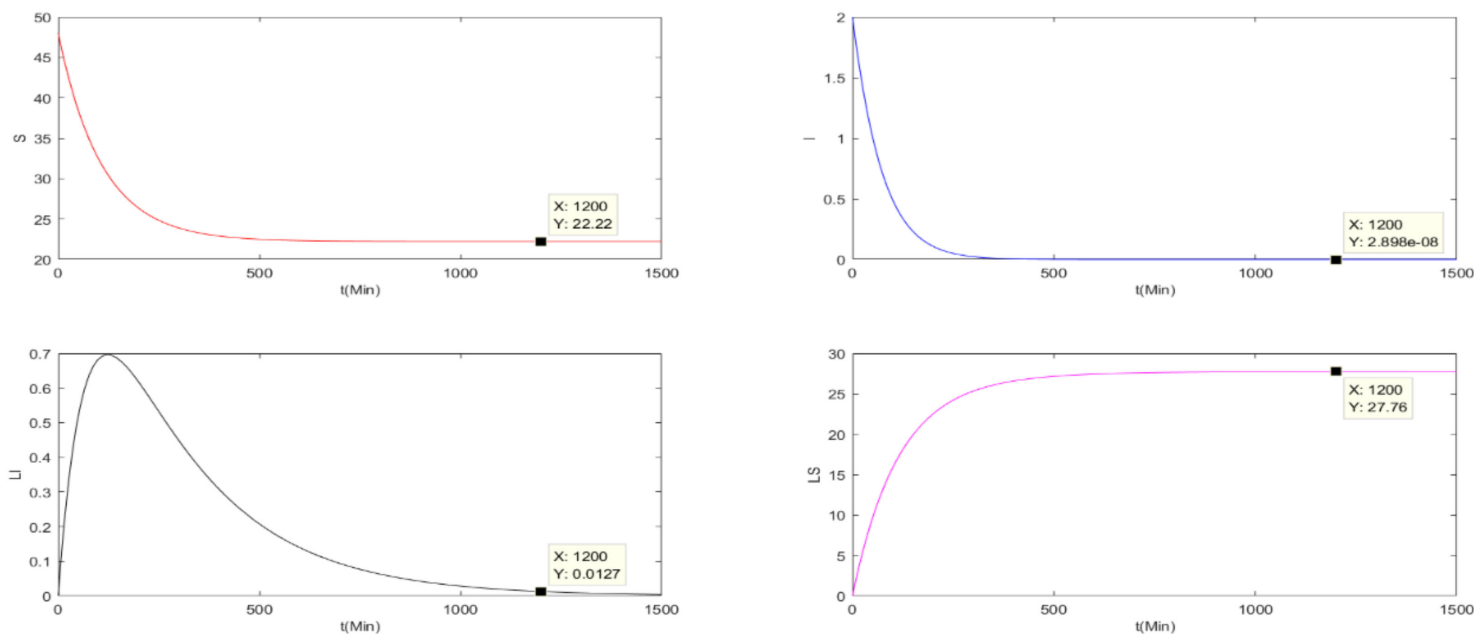

Figure 3. The nodes' numbers under the non-charging model with $R_{2}<1$. 


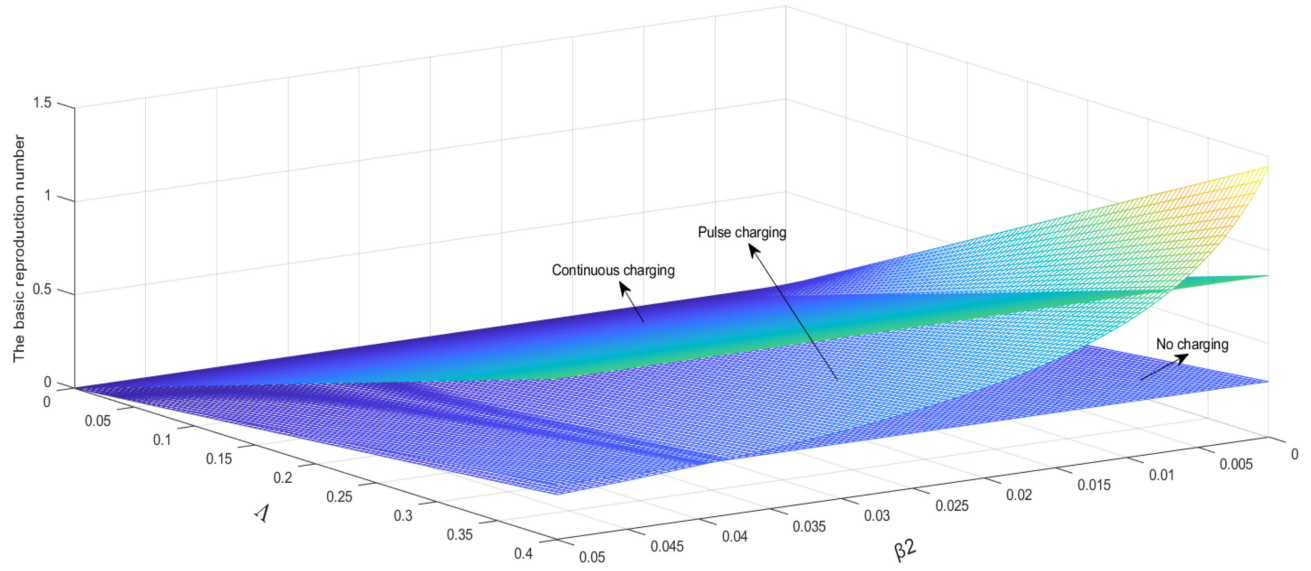

Figure 4. $\Lambda$ and $\beta_{2}$ relationships with the basic reproduction number.

A set of parameters is set as $\Lambda=0.2, \mu=0.004, \beta_{1}=0.005, \beta_{3}=0.008, \alpha_{2}=0.001$, $\gamma=0.05, a=0.005, T=10, \beta_{2} \in[0,0.05], \alpha_{1} \in[0,1]$. As shown in Figure 5 , it is obvious that the increase in $\alpha_{1}$ increases the basic reproduction number more significantly than that of $\Lambda$, and $\beta_{2}$ the influence on the basic reproduction number is almost the same as the change in Figure 4. Thereby determining that $\beta_{2}$ only has a significant impact on the pulsed charging model.

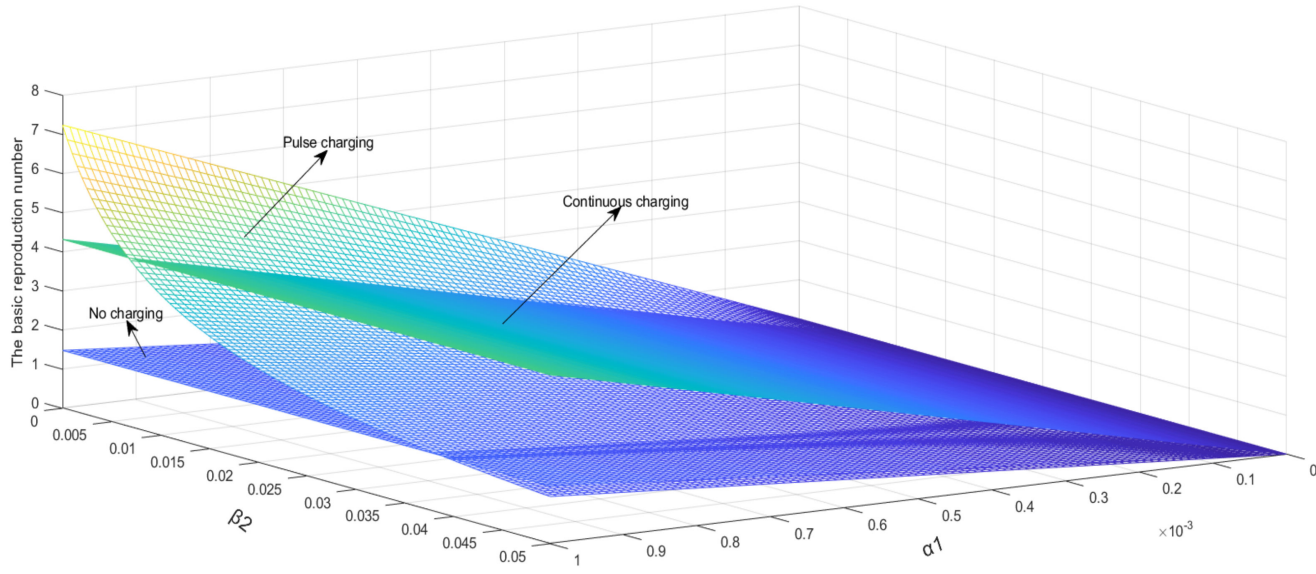

Figure 5. $\alpha_{1}$ and $\beta_{2}$ relationships with the basic reproduction number.

A set of parameters is set as $\Lambda=0.2, \mu=0.004, \beta_{1}=0.005, \beta_{2}=0.005, \beta_{3}=0.008$, $\alpha_{1}=0.0001, \alpha_{2}=0.001, T=10, \gamma \in[0,0.05], a \in[0,0.1]$. As shown in Figure 6, the basic reproduction number will decrease with the increase in $a$, and the basic reproduction number will increase with the increase in $\gamma$. It is obvious that $a$ has greater influence than $\gamma$ on the basic reproduction number.

\subsection{Sensitivity Analysis}

In this section, we carefully study the sensitivity of the SIALILS model threshold by evaluating PRCC (partial correlation coefficient). Evenly distributed sampling is carried out for each input parameter of the model, where the maximum value of each parameter is $120 \%$ of the sampling baseline value, and the minimum value is $80 \%$ of the baseline value. As we know, when the absolute value of PRCC is less than 0.2 , the correlation between input parameters and output variables is not significant [43], the absolute value is moderately correlated between 0.2 and 0.4 and highly correlated when the absolute value is greater than 0.4. The distribution interval of each parameter and its corresponding PRCC are shown in Table 3. 


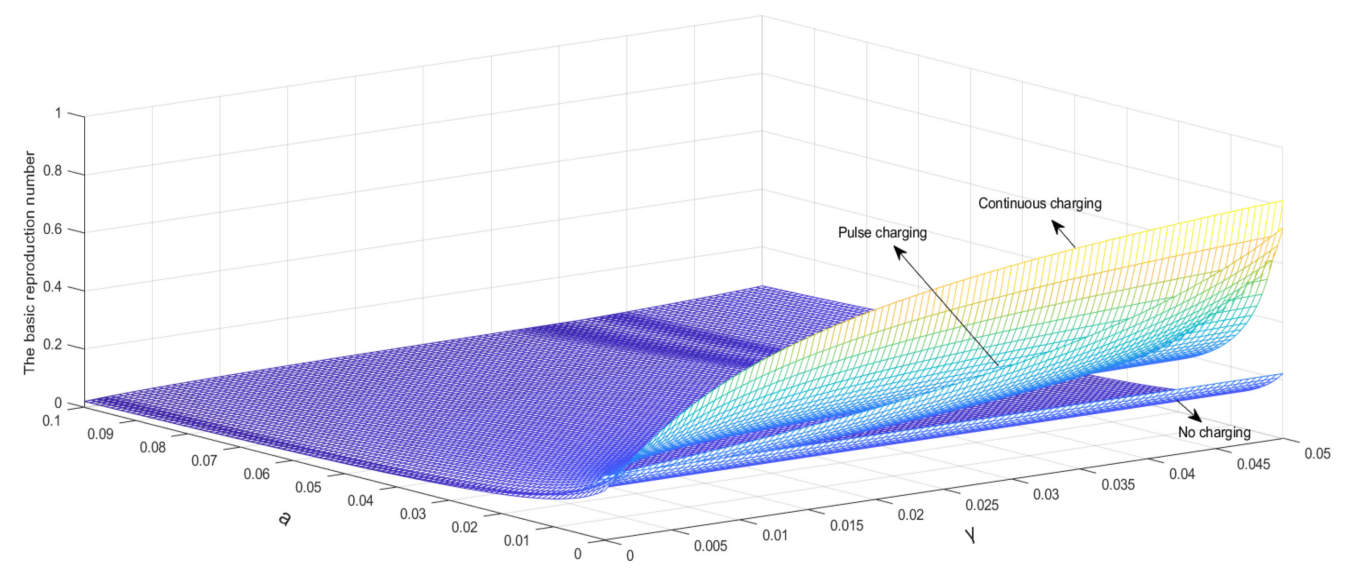

Figure 6. $a$ and $\gamma$ relationships with the basic reproduction number.

Table 3. PRCCs values.

\begin{tabular}{ccc}
\hline Parameters & Range & PRCC \\
\hline$\lambda$ & $\mathrm{U}(0.32,0.48)$ & 0.9515 \\
$\mu$ & $\mathrm{U}(0.24,0.36)$ & -0.9583 \\
$\beta_{1}$ & $\mathrm{U}(0.032,0.048)$ & -0.5192 \\
$\beta_{2}$ & $\mathrm{U}(0.032,0.048)$ & -0.5406 \\
$\beta_{3}$ & $\mathrm{U}(0.0004,0.0005)$ & 0.0136 \\
$\alpha_{1}$ & $\mathrm{U}(0.0032,0.0048)$ & 0.9524 \\
$\alpha_{2}$ & $\mathrm{U}(0.008,0.012)$ & -0.1737 \\
$\gamma$ & $\mathrm{U}(0.12,0.18)$ & -0.5593 \\
$a$ & $\mathrm{U}(0.004,0.006)$ & 0.0263 \\
\hline
\end{tabular}

From Figure 7, it is obvious that the birth rate $\lambda$ and metastasis rate of $\alpha_{1}$ are related to the threshold $R_{0}$. However, death rate $\mu$, metastasis rate $\beta_{1}, \beta_{2}$ and $\gamma$ are correlated with threshold $R_{0}$ is significantly negatively correlated, and the remaining parameters are not correlated. This means that if we want to lower the threshold $R_{0}$, we can lower the birth rate $\lambda$ and metastasis rate $\alpha_{1}$, or increase the death rate $\mu$, transfer rate $\beta_{1}, \beta_{2}$ and $\gamma$. Conversely, we can increase the threshold $R_{0}$ by increasing the birth rate $\lambda$ and metastasis rate $\alpha_{1}$, or decreasing mortality rate $\mu$, metastasis rate $\beta_{1}, \beta_{2}$ and $\gamma$. From Figure 8 , it can be easily learned that within the range of parameters specified by us, the threshold $R_{0}$ is only between 0.1 and 0.35 , and most of them are between 0.15 and 0.25 . It can be seen that within this range, the threshold $R_{0}$ have limited changes. 


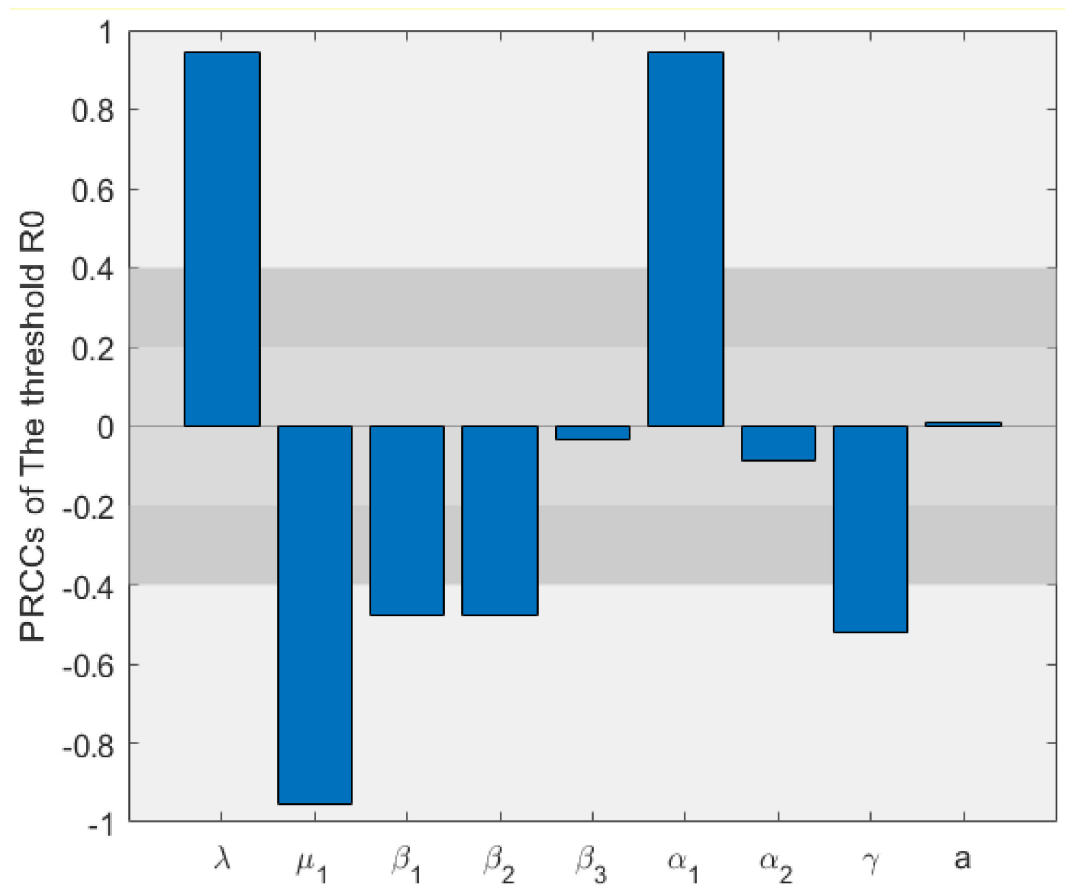

Figure 7. PRCCs of $R_{0}$.

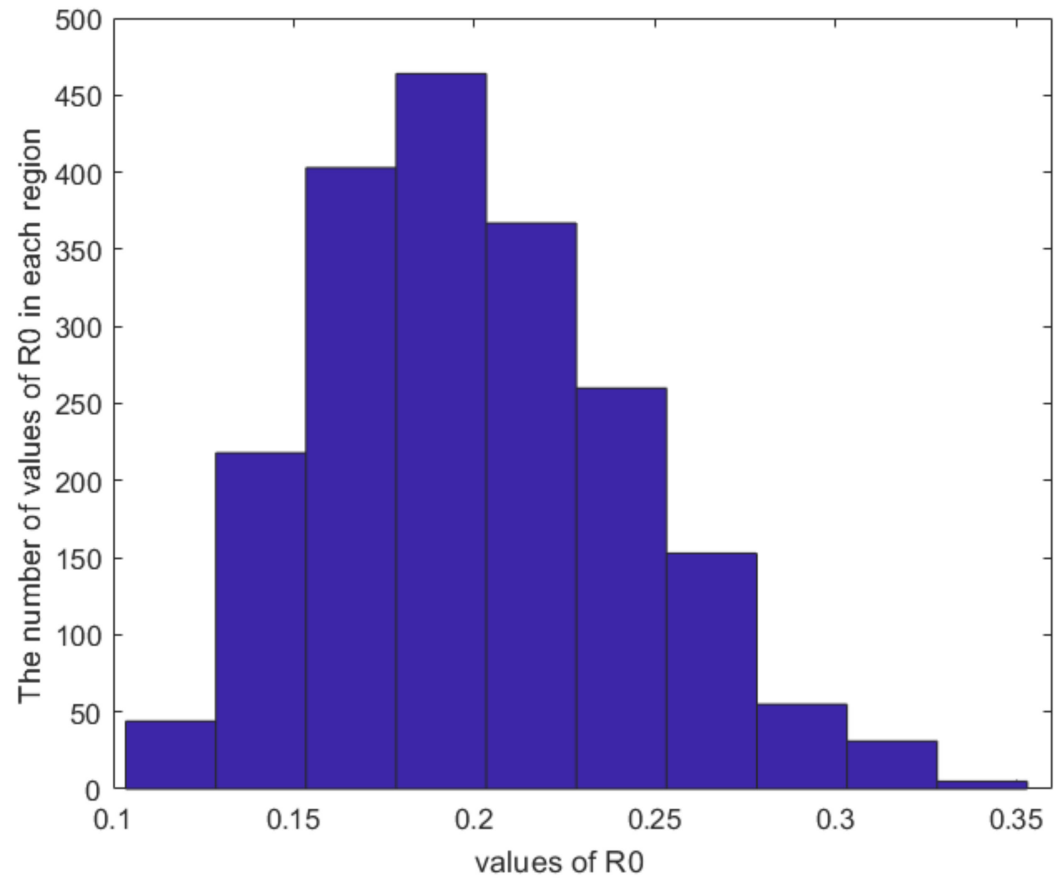

Figure 8. The distribution of the values of $R_{0}$.

\subsection{Optimal Control Strategy}

In this section, considering the treatment cost of infected nodes, control factors are designed. Four control strategies (MAX control, MIN control, average control, optimal control) are applied to the pulse charging model, and the optimal control problem is numerically simulated. The superiority and effectiveness of the optimal control strategy are verified by comparing the number of nodes in each state and the control cost. Parameters of the four control policies are set as shown in Table 4 . The parameters and weight parameters settings of the proposed model are shown in Table 5, where $A_{1}, A_{2}, A_{3}$ and $B$ are the weight parameters. 
Table 4. Parameters setting of four control strategies.

\begin{tabular}{|c|c|c|c|c|}
\hline Case & Control Strategy & $\alpha_{2}$ & $\mathbf{v}$ & $\mathbf{J}$ \\
\hline 1 & Optimal control & $\alpha_{2}^{*}(t)$ & $v^{*}(n T)$ & \multirow{4}{*}{$\begin{array}{c}\int_{0}^{\delta T}\left(A_{1} I(t)+A_{2} L I(t)+\right. \\
\left.\frac{A_{3}}{2} \alpha_{2}^{2}(t)\right) \mathrm{d} t+ \\
\sum_{i=1}^{\eta}\left(\frac{B}{2} V^{2}(n T)\right)\end{array}$} \\
\hline 2 & MIN control & 0 & 0 & \\
\hline 3 & MAX control & 1 & 1 & \\
\hline 4 & AVERAGE = control & $\alpha_{2}^{*}(t)=\frac{\sum_{0}^{T} \alpha_{2}^{*}(t)}{T}=0.5720$ & $v^{*}(n T)=\frac{\sum_{1}^{\delta} v^{*}(n T)}{\delta}=0.0587$ & \\
\hline
\end{tabular}

Table 5. Parameters setting.

\begin{tabular}{cccc}
\hline Notation & Value & Notation & Value \\
\hline$\lambda$ & 0.4 & $\mathrm{a}$ & 0.005 \\
$\mu$ & 0.04 & $\mathrm{~T}$ & 4 \\
$\beta_{1}$ & 0.05 & $A_{1}$ & 0.6 \\
$\beta_{2}$ & 0.05 & $A_{2}$ & 0.01 \\
$\beta_{3}$ & 0.005 & $A_{3}$ & 0.05 \\
$\alpha_{1}$ & 0.03 & $B$ & 0.05 \\
$\gamma$ & 0.2 & & \\
\hline
\end{tabular}

We set the initial node number as $S_{0}=40, I_{0}=10, A_{0}=0, L S_{0}=0$ and $L I_{0}=0$. The control time is set as 20 days. In Figure 9, the change of the number of nodes of each control strategy over time is shown. Under the MAX control, $v(n T)=1$, the number of $I$ nodes will change greatly at the pulse time to reduce the number of $I$ nodes and increase the number of S nodes so that the model can operate efficiently. In MIN control, $v(n T)=0$, the number of $I$ nodes will greatly increase at the pulse time, and the number of $L I$ nodes will also increase accordingly. Under this control, the number of $A$ nodes is always 0 , resulting in the failure of anti-malware $A$ to run normally; the number of $S$ and $L S$ nodes will decrease rapidly. Obviously, MIN control strategy finds it difficult to suppress the spread of malware, leading to the normal operation of anti-malware $A$, and the number of $S$ and LS nodes will decrease rapidly. Obviously, the MIN control strategy finds it difficult to suppress the spread of malware. On the whole, the number of the $S$ node decreases most obviously under the minimum control strategy, while the number of the I node is completely opposite. The rising rate of the number of the I node under the MIN control strategy is far higher than that of the other three control strategies. It can determine that the MIN control strategy control effect is poorer and the spread of the virus is difficult to control. In the case of the four control strategies, regardless of cost, the Max control strategy effect is the best because it can maintain the high-energy operation of the $S$ nodes and rapidly reduce the number of I nodes to make the system run efficiently. The control effect of the optimal control strategy follows closely.

Then, the number of nodes in the model under the four control strategies is further analyzed. According to Figure 10, it can be seen that the number of $I$ nodes rapidly decreases to 0 in optimal control, max control and average control, and the number of $L I$ nodes hardly changes. However, under min control, the number of I nodes occupy the majority, and the number of $L I$ nodes and increases accordingly. It is surprising that the number of nodes under optimal control is almost the same as that under max control, which further verifies the superiority of the optimal control strategy.

Finally, the cost of the four control strategies is numerically simulated. It is obvious from Figure 11 that min control has the highest cost, while the optimal control has the lowest cost. The cost of the four control strategies is compared as follows: Cost $\min _{\text {in }}>$ Cost $_{\max }>$ $\operatorname{Cost}_{\text {AVERAGE }}>\operatorname{Cost}_{\text {OPTIMAL }}$. The effectiveness of the optimal control is verified. 

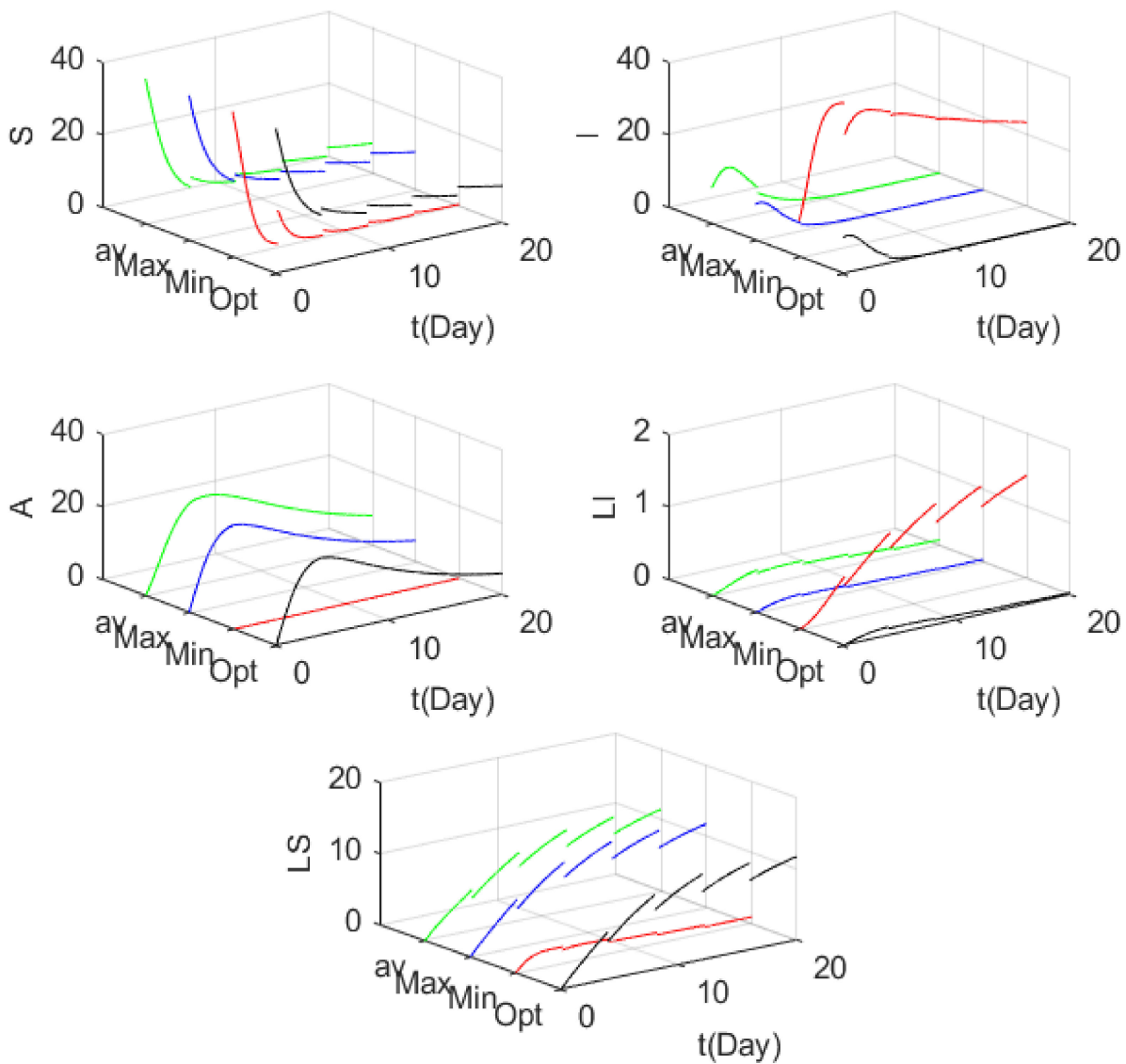

Figure 9. The number of nodes changes with time under the four control strategies.
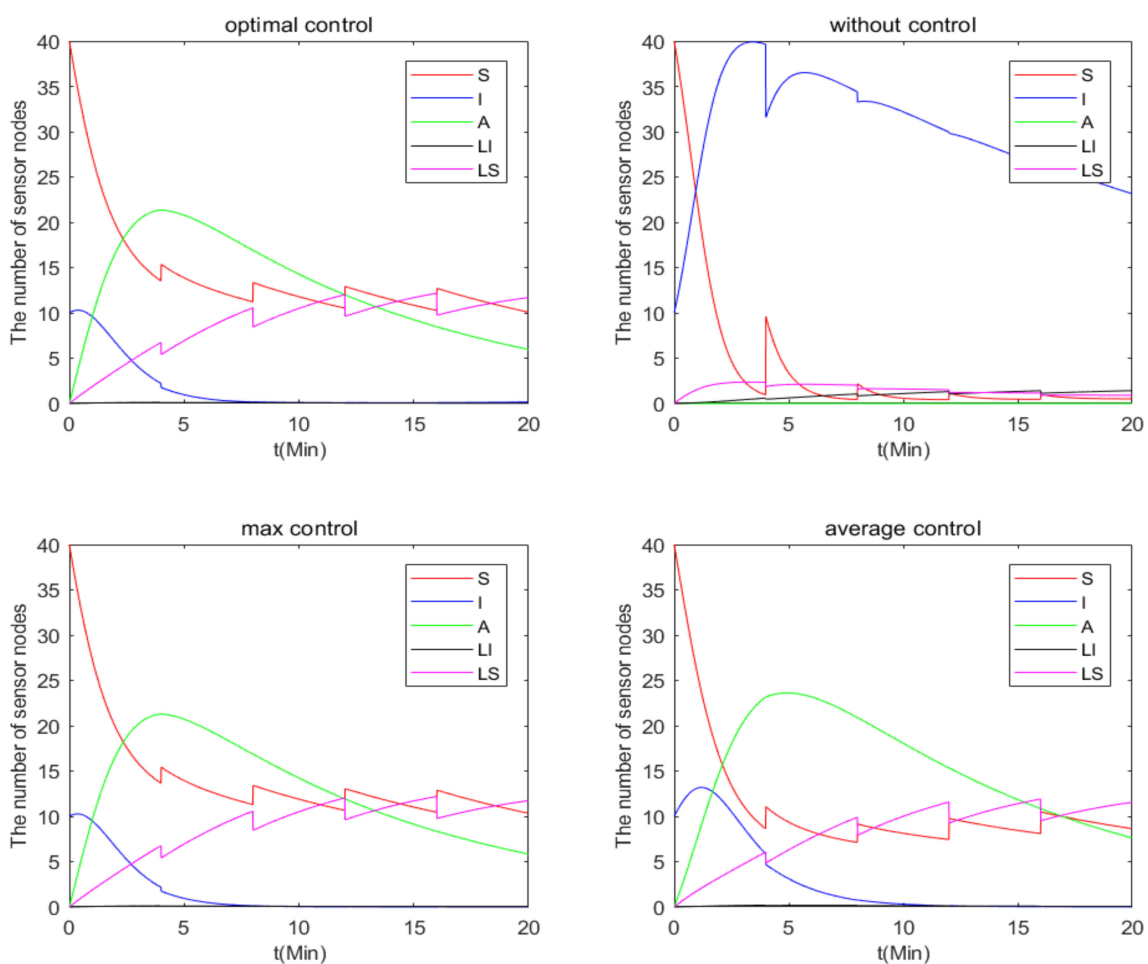

Figure 10. The number of nodes of the four control strategies. 


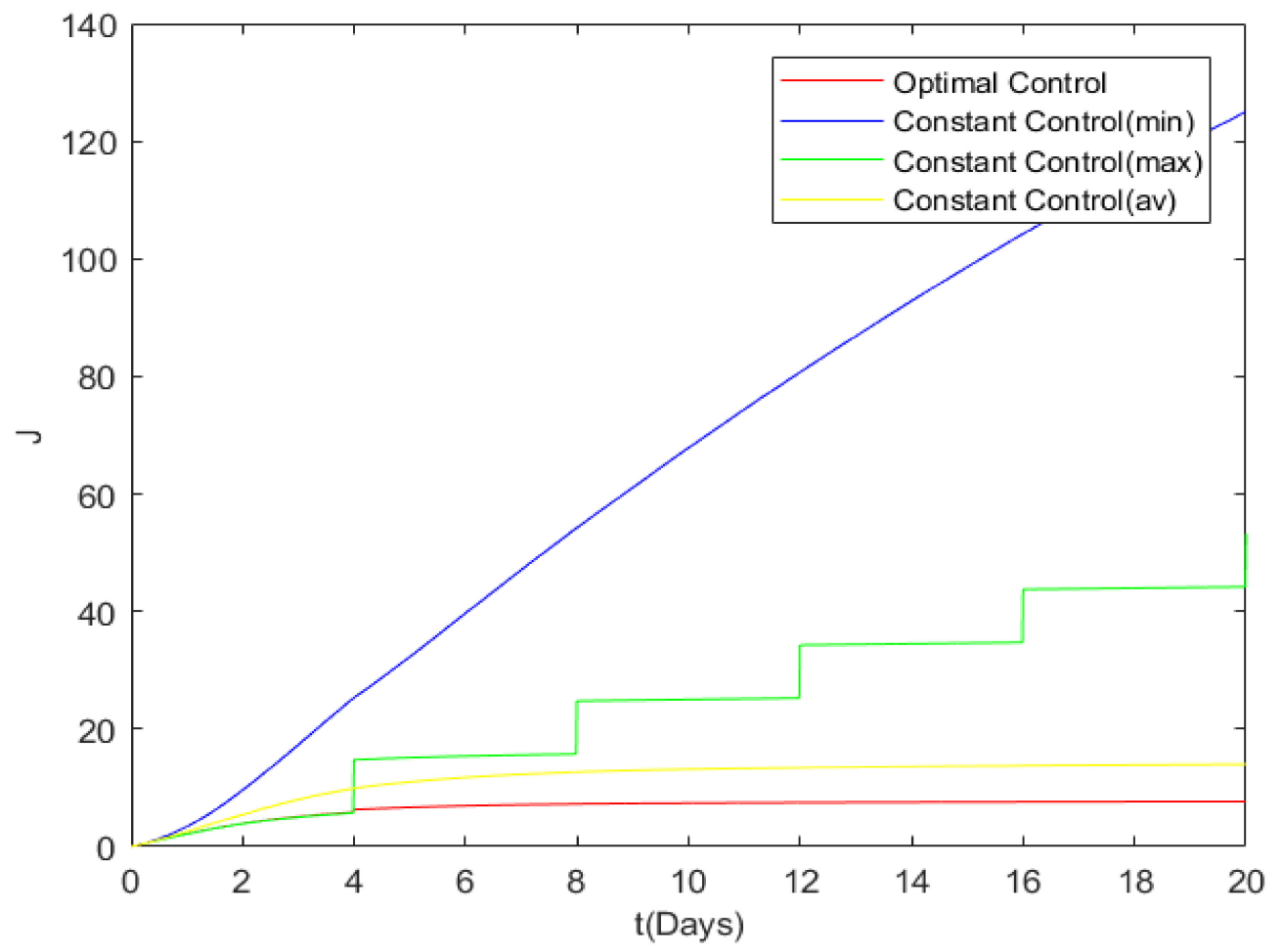

Figure 11. Cost comparison of the four control strategies.

\section{Conclusions}

In this paper, a novel model of the epidemic based on pulse charging (SIALS-P) for WRSNs is proposed. In each periodic pulse point, the low energy states (LS nodes, LI nodes) are converted into the normal energy states ( $S$ nodes, I nodes). The stability of the model is analyzed, and the local and global stability of the malware-free $T$-period solution is also proven. Additionally, the comparison theorem is used to prove the persistence of malware transmission. In order to reduce the model control cost, we propose an optimal control strategy for the proposed model based on the Pontryagin maximum principle. Finally, in the numerical simulation part, we use the Runge-Kutta method to further verify the correctness of the theory and compare the model with the non-charging and continuous charging models. The simulation results show that the number of nodes in pulse charging mode is between those in the other two modes. This suggests that the pulse charging model is more energy-saving when compared with the continuous charging model and has higher working efficiency compared with the non-charging model. The influence of each parameter on the basic reproduction number is given by simulation. PRCC is used to analyze the sensitivity of threshold parameter $R_{0}$ and the feasibility and superiority of optimal control are further verified.

Of course, the study in this paper also has limitations. For example, the simulation results of its periodic or chaotic solutions are unknown. In addition, if a node has an incubation period after infection, it can lead to more complex system behavior (for example, repeated outbreaks, etc.).

Author Contributions: Conceptualization, G.L., X.Z. and Z.L.; methodology, X.S., F.H. and X.W.; software, X.S. and Z.H.; validation, X.S. and F.H.; formal analysis, X.S. and F.H.; investigation, G.L., X.S., F.H., Z.L., X.Z., X.W. and Z.H.; resources, G.L. and X.S.; data curation, X.S. and F.H.; writingoriginal draft preparation, X.S. and F.H.; writing-review and editing, X.S. and F.H.; visualization, X.S.; supervision, G.L.; funding acquisition, G.L. All authors have read and agreed to the published version of the manuscript.

Funding: This research was funded by National Natural Science Foundation of China (51975136, 61403089), Guangzhou University Research Project (YJ2021002); the 2020 Department of Education of 
Guangdong Province Innovative and Strong School Project (Natural Sciences)-Young Innovators Project (Natural Sciences) under Grant 2020KQNCX054; the National Key Research and Development Program of China (2018YFB2000501); the Science and Technology Innovative Research Team Program in Higher Educational Universities of Guangdong Province (2017KCXTD025); the Innovative Academic Team Project of Guangzhou Education System (1201610013); the Special Research Projects in the Key Fields of Guangdong Higher Educational Universities (2019KZDZX1009); the Science and Technology Research Project of Guangdong Province (2017A010102014, 2016A010102022); the Guangzhou University Research Project (YJ2021002); and the Science and Technology Research Project of Guangzhou (201707010293).

Institutional Review Board Statement: Not applicable.

Informed Consent Statement: Not applicable.

Data Availability Statement: Not applicable.

Acknowledgments: The authors acknowledge funding received from the science foundations. They are appreciated for supporting this work.

Conflicts of Interest: The authors declare no conflict of interest.

\section{References}

1. Xie, H.; Yan, Z.; Yao, Z.; Atiquzzaman, M. Data collection for security measurement in wireless sensor networks: A survey. IEEE Internet Things J. 2019, 6, 2205-2224. [CrossRef]

2. Han, G.; Jiang, J.; Zhang, C.; Duong, T.Q.; Guizani, M.; Karagiannidis, G.K. A survey on mobile anchor node assisted localization in wireless sensor networks. IEEE Commun. Surv. Tutor. 2016, 18, 2220-2243. [CrossRef]

3. Butun, I.; Osterberg, P.; Song, H. Security of the internet of things: Vulnerabilities, attacks, and countermeasures. IEEE Commun. Surv. Tutor. 2020, 22, 616-644. [CrossRef]

4. Sajan, R.I.; Jasper, J. A secure routing scheme to mitigate attack in wireless adhoc sensor network. Comput. Secur. 2021, $103,102197$.

5. Ge, C.; Yin, C.; Liu, Z.; Fang, L.; Zhu, J.; Ling, H. A privacy preserve big data analysis system for wearable wireless sensor network. Comput. Secur. 2020, 96, 101887. [CrossRef]

6. Rashid, B.; Rehmani, M.H. Applications of wireless sensor networks for urban areas: A survey. J. Netw. Comput. Appl. 2016, 60, 192-219. [CrossRef]

7. Yetgin, H.; Cheung, K.T.K.; El-Hajjar, M.; Hanzo, L.H. A Survey of network lifetime maximization techniques in wireless sensor networks. IEEE Commun. Surv. Tutor. 2017, 19, 828-854. [CrossRef]

8. Panatik, K.Z.; Kamardin, K.; Shariff, S.A.; Yuhaniz, S.S.; Ahmad, N.A.; Yusop, O.M.; Ismail, S. Energy harvesting in wireless sensor networks: A survey. In Proceedings of the 2016 IEEE 3rd International Symposium on Telecommunication Technologies, Kuala Lumpur, Malaysia, 28-30 November 2016; pp. 53-58.

9. Shu, Y.; Yousefi, H.; Cheng, P.; Chen, J.; Gu, Y.J.; He, T.; Shin, K.G. Near-optimal velocity control for mobile charging in wireless rechargeable sensor networks. IEEE Trans. Mob. Comput. 2016, 15, 1699-1713. [CrossRef]

10. Wu, P.; Xiao, F.; Sha, C.; Huang, H.; Sun, L. Trajectory optimization for UAVs' efficient charging in wireless rechargeable sensor networks. IEEE Trans. Veh. Technol. 2020, 69, 4207-4220. [CrossRef]

11. Mo, L.; Kritikakou, A.; He, S. Energy-aware multiple mobile chargers coordination for wireless rechargeable sensor networks. IEEE Internet Things J. 2019, 6, 8202-8214. [CrossRef]

12. Piqueira, J.R.C.; Cabrera, M.A.M.; Batistela, C.M. Malware propagation in clustered computer networks. Phys. A Stat. Mech. Its Appl. 2021, 573, 125958. [CrossRef]

13. Guillén, J.H.; del Rey, A.M. A mathematical model for malware spread on WSNs with population dynamics. Phys. A Stat. Mech. Its Appl. 2020, 545, 123609. [CrossRef]

14. Huang, D.-W.; Yang, L.-X.; Yang, X.; Wu, Y.; Tang, Y.Y. Towards understanding the effectiveness of patch injection. Phys. A Stat. Mech. Its Appl. 2019, 526, 120956. [CrossRef]

15. Liu, G.; Peng, B.; Zhong, X. A novel epidemic model for wireless rechargeable sensor network security. Sensors 2021, $21,123$. [CrossRef]

16. Liu, G.; Huang, Z.; Wu, X.; Liang, Z.; Hong, F.; Su, X. Modelling and analysis of the epidemic model under pulse charging in wireless rechargeable sensor networks. Entropy 2021, 23, 927. [CrossRef]

17. Liu, G.; Li, J.; Liang, Z.; Peng, Z. Analysis of time-delay epidemic model in rechargeable wireless sensor networks. Mathematics 2021, 9, 978. [CrossRef]

18. Liu, G.; Li, J.; Liang, Z.; Peng, Z. Dynamical behavior analysis of a time-delay SIRS-L model in rechargeable wireless sensor networks. Mathematics 2021, 9, 2007. [CrossRef]

19. Liu, G.; Peng, B.; Zhong, X. Epidemic analysis of wireless rechargeable sensor networks based on an attack-defense game model. Sensors 2021, 21, 594. [CrossRef] 
20. Liu, G.; Peng, B.; Zhong, X.; Cheng, L.; Li, Z. Attack-defense game between malicious programs and energy-harvesting wireless sensor networks based on epidemic modeling. Complexity 2020, 2020, 3680518. [CrossRef]

21. Liu, G.; Peng, B.; Zhong, X.; Lan, X. Differential games of rechargeable wireless sensor networks against malicious programs based on SILRD propagation model. Complexity 2020, 2020, 5686413. [CrossRef]

22. Liu, G.; Peng, Z.; Liang, Z.; Li, J.; Cheng, L. Dynamics analysis of a wireless rechargeable sensor network for virus mutation spreading. Entropy 2021, 23, 572. [CrossRef] [PubMed]

23. Shen, S.; Zhou, H.; Feng, S.; Liu, J.; Zhang, H.; Cao, Q. An epidemiology-based model for disclosing dynamics of malware propagation in heterogeneous and mobile WSNs. IEEE Access 2020, 8, 43876-43887. [CrossRef]

24. Ojha, R.P.; Srivastava, P.K.; Sanyal, G.; Gupta, N. Improved model for the stability analysis of wireless sensor network against malware attacks. Wirel. Pers. Commun. 2021, 116, 2525-2548. [CrossRef]

25. Hosseini, S.; Azgomi, M.A. The dynamics of an SEIRS-QV malware propagation model in heterogeneous networks. Phys. A Stat. Mech. Its Appl. 2018, 512, 803-817. [CrossRef]

26. D'Onofrio, A. Stability properties of pulse vaccination strategy in SEIR epidemic model. Math. Biosci. 2002, 179, 57-72. [CrossRef]

27. Zhang, D.; Zhang, Y.; Xu, B.; Yang, Z. Pulse vaccination control of an SIR epidemic model with distributed delays. Adv. Appl. Math. 2020, 9, 359-365. [CrossRef]

28. Zhou, A.; Sattayatham, P.; Jiao, J. Dynamics of an SIR epidemic model with stage structure and pulse vaccination. Adv. Differ. Equ. 2016, 2016, 700. [CrossRef]

29. Yu, D.; Gao, S.; Luo, Y.; Xie, F. Pulse vaccination strategy in an SIVS epidemic model with general nonlinear incidence rate. Adv. Anal. 2016, 1, 80-88. [CrossRef]

30. Wang, S.; Li, D.; Wu, Y. Stability analysis of a SEIQR epidemic model with pulse vaccination. J. Harbin Univ. Sci. Technol. 2017, $22,72-77$.

31. Zhao, Z.; Pang, L.; Chen, Y. Nonsynchronous bifurcation of SIRS epidemic model with birth pulse and pulse vaccination. Nonlinear Dyn. 2015, 79, 2371-2383. [CrossRef]

32. Wang, X.; He, Z.; Zhang, L. A pulse immunization model for inhibiting malware propagation in mobile wireless sensor networks. Chin. J. Electron. 2014, 23, 810-815.

33. Al Kindi, A.; Al Abri, D.; Al Maashri, A.; Bait-Shiginah, F. Analysis of malware propagation behavior in Social Internet of Things. Int. J. Commun. Syst. 2019, 32, e4102. [CrossRef]

34. Gao, S.J.; Zhong, D.M.; Zhang, Y. Analysis of novel stochastic switched SILI epidemic models with continuous and impulsive control. Phys. A Stat. Mech. Its Appl. 2018, 495, 162-171. [CrossRef]

35. Srivastava, P.K.; Pandey, S.P.; Gupta, N.; Singh, S.P.; Ojha, R.P. Modeling and analysis of antimalware effect on wireless sensor network. In Proceedings of the IEEE 4th International Conference on Computer and Communication Systems, Singapore, 23-25 February 2019; pp. 639-643. [CrossRef]

36. Ma, Z.; Zhou, Y.; Wu, J. Modeling and Dynamics of Infectious Diseases; World Scientific Publishing Company: Singapore, 2009. [CrossRef]

37. Gao, J.-Z.; Zhang, T.-L. An SIRS epidemic model with pulse vaccination, birth pulse and logistic death rate. Commun. Math. Res. 2019, 35, 247-263.

38. Verhulst, F. Nonlinear Differential Equations and Dynamical Systems; Springer: Berlin/Heidelberg, Germany, 1990.

39. Kulev, G.; Bainov, D. Application of lyapunov's direct method to the investigation of the global stability of the solutions of systems with impulse effect. Appl. Anal. 1988, 26, 255-270. [CrossRef]

40. Yin, Y.; Cao, Y.; Yao, L.-Q. A new meshless method based on 2D-EFG for the analysis of piezoelectric laminated Timoshenko beam. In Proceedings of the Symposium on Piezoelectricity, Acoustic Waves and Device Applications (SPAWDA), Shenzhen, China, 9-11 December 2011. [CrossRef]

41. Abouelkheir, I.; El Kihal, F.; Rachik, M.; Elmouki, I. Optimal impulse vaccination approach for an SIR control model with short-term immunity. Mathematics 2019, 7, 420. [CrossRef]

42. Zhang, G.; Song, M. Asymptotical stability of Runge-Kutta methods for advanced linear impulsive differential equations with piecewise constant arguments. Appl. Math. Comput. 2015, 259, 831-837. [CrossRef]

43. Zhang, Y.H.; Xiao, Y.N. Modeling and analyzing the effects of fixed-time intervention on transmission dynamics of echinococcosis in Qinghai province. Math. Methods Appl. Sci. 2021, 44, 4276-4296. [CrossRef] 